\title{
Clinical Efficacy and Safety of Antiviral Drugs in the Extended Use against COVID-19: What We Know So Far
}

\author{
Md. Jamal Hossain ${ }^{1, *}{ }^{\oplus}$, Tabassum Jannat ${ }^{1, \dagger}{ }^{+}$, Shejuti Rahman Brishty ${ }^{2,+}{ }^{\oplus}$, Urmi Roy $^{3, \dagger}$, Saikat Mitra ${ }^{4}$, \\ Md. Oliullah Rafi ${ }^{5}{ }^{(}$, Md. Rabiul Islam ${ }^{2}{ }^{-}$, Mst. Luthfun Nesa ${ }^{1}$, Md. Ariful Islam ${ }^{6}$ and Talha Bin Emran ${ }^{7, *(\mathbb{B})}$ \\ 1 Department of Pharmacy, State University of Bangladesh, 77 Satmasjid Road, Dhanmondi, \\ Dhaka 1205, Bangladesh; tabassum_jannat@uap-bd.edu (T.J.); luthfun@sub.edu.bd (M.L.N.) \\ 2 Department of Pharmacy, University of Asia Pacific, 74/A, Green Road, Farmgate, Dhaka 1205, Bangladesh; \\ shejutirahman846@gmail.com (S.R.B.); robi.ayaan@gmail.com (M.R.I.) \\ 3 Department of Pharmacy, Stamford University Bangladesh, 51 Siddeswari Road, Ramna, Dhaka 1217, Bangladesh; \\ urmilaurmi61@gmail.com \\ 4 Department of Pharmacy, Faculty of Pharmacy, University of Dhaka, Dhaka 1000, Bangladesh; \\ saikatmitradu@gmail.com \\ 5 Department of Genetic Engineering and Biotechnology, Jashore University of Science and Technology, \\ Jashore 7408, Bangladesh; rafi.btech.bd@gmail.com \\ 6 Bangladesh Reference Institute for Chemical Measurements, Dr. Qudrat-e-Khuda Road, Dhanmondi, \\ Dhaka 1205, Bangladesh; islam.ma.phr@gmail.com \\ 7 Department of Pharmacy, BGC Trust University Bangladesh, Chittagong 4381, Bangladesh \\ * Correspondence: jamal.du.p48@gmail.com (M.J.H.); talhabmb@bgctub.ac.bd (T.B.E.); \\ Tel.: +88-015-1781-4866 (M.J.H.); +88-018-1994-2214 (T.B.E.) \\ + These authors contributed equally to this work.
}

Brishty, S.R.; Roy, U.; Mitra, S.; Rafi, M.O.; Islam, M.R.; Nesa, M.L.; Islam, M.A.; Emran, T.B. Clinical Efficacy and Safety of Antiviral Drugs in the Extended Use against COVID-19: What We Know So Far. Biologics 2021, 1, 252-284. https://doi.org/10.3390/ biologics1020016

Academic Editors: Vasso Apostolopoulos and

Majid Hassanzadeganroudsari

Received: 27 July 2021

Accepted: 13 September 2021

Published: 15 September 2021

Publisher's Note: MDPI stays neutral with regard to jurisdictional claims in published maps and institutional affiliations.

\begin{abstract}
Human beings around the globe have been suffering from a devastating novel pandemic and public health emergency, coronavirus disease 2019 (COVID-19), for more than one and a half years due to the deadly and highly pathogenic severe acute respiratory coronavirus 2 (SARS-CoV-2) infection worldwide. Notably, no effective treatment strategy has been approved for the complete recovery of COVID-19 patients, though several vaccines have been rolled out around the world upon emergency use authorization. After the emergence of the COVID-19 outbreak globally, plenty of clinical investigations commenced to screen the safety and efficacy of several previously approved drugs to be repurposed against the SARS-CoV-2 pathogen. This concise review aims at exploring the current status of the clinical efficacy and safety profile of several antiviral medications for the treatment of patients with COVID-19 and other respiratory complications caused by SARS-CoV-2 infection. The paper covers all kinds of human studies (January 2020 to June 2021) except case reports/series to highlight the clear conclusion based on the current clinical evidence. Among the promising repositioned antivirals, remdesivir has been recommended in critical conditions to mitigate the fatality rate and improve clinical conditions. In addition, boosting the immune system is believed to be beneficial in treating COVID-19 patients, so interferon type I might exert immunomodulation through its antiviral effects by stimulating interferon-stimulated gene (ISG). However, more extensive clinical studies covering all ethnic groups globally are warranted based on current data to better understand the clinical efficacy of the currently proposed repurposed drugs against COVID-19.
\end{abstract}

Keywords: coronavirus disease 2019 (COVID-19); repurposing strategy; repurposed drugs; remdesivir; interferon type I; clinical trials

Copyright: (c) 2021 by the authors. Licensee MDPI, Basel, Switzerland. This article is an open access article distributed under the terms and conditions of the Creative Commons Attribution (CC BY) license (https:// creativecommons.org/licenses/by/ $4.0 /)$.

\section{Introduction}

The outbreak of coronavirus disease 2019 (COVID-19) has created a lot of burden on the global medical system, public health, and economic and social life of human beings [1,2]. The causative pathogen identified for COVID-19 is called severe acute respiratory syndrome coronavirus 2 (SARS-CoV-2), which emerged from viruses of unknown sources [3,4]. 
SARS-CoV- 2 is a type of $\beta$-coronavirus ( $\beta-\mathrm{CoV})$ that belongs to the coronavirus group. The human coronavirus group causes several outbreaks, including the severe acute respiratory syndrome (SARS-CoV) epidemic from 2002 to 2004 and the Middle East respiratory syndrome (MERS-CoV) outbreak in the Middle East, Africa, and South Asia, and many more countries during $2012[5,6]$. Wuhan, China, was the first city for the occurrence of COVID-19, and after that, it spread all over the world, infecting around 218 million people together with over 4.5 million total deaths, according to the COVID-19 global case dashboard of the World Health Organization (WHO), by 29 August 2021 [7]. Based on the data from January 2020, the WHO declared COVID-19 a Public Health Emergency of International Concern (PHEIC), which was followed by a pandemic on 11 March 2020 [8].

In the meantime, many vaccines have received emergency use authorization, and by 30 August 2021, 5.24 billion doses had been administered globally. Around $40 \%$ of the world population has received at least one dose of the COVID-19 vaccine. It is notable that approximately 39 million people are taking the COVID-19 vaccine per day. However, only $1.6 \%$ of people from low-income countries have received at least one dose of the vaccine to be inoculated [9]. In addition, the production and equitable distribution of a large number of vaccine doses is challenging for world leaders to achieve within a short time. Another concern is that political and financial matters might be a significant barrier to administering the vaccines to the entire world population for all ethnic and socioeconomic groups. The development and production of the vaccines within a very narrow time frame might have implied insufficient evidence for the safety and efficacy of the vaccines in the long term [10]. Therefore, the world community must develop new effective and safe antiviral drugs or therapeutics to fight against COVID-19.

To date, no effective and approved antiviral treatment is available to fight against COVID-19. However, some recommendations are being practiced concurrently to manage individual patients' needs, such as antipyretic drugs for fever, oxygen therapy for respiratory distresses, antimicrobial therapy with mechanical ventilation applied in some severe cases depending on the clinical condition of the patient, and so on [11,12]. Meanwhile, the drug repurposing strategy is being continuously used in COVID-19 treatment. Drug repurposing or drug repositioning is tactically a rapid process to identify new pharmacological indications rather than the original purpose of investigational, existing, already marketed or FDA-approved drugs for the treatment of diseases. This advantageous method provides a great benefit in circumventing some de novo drug design and development stages. Thus, the technique decreases scheduled periods of drug development, reduces failure risk, and protects funds from being wasted [13]. In addition, it is crucial for a drug to be effective, proportionally related to clinical efficacy, or to produce desired pharmacological activities for a specified indication in humans. A potential drug must be passed through efficacy trials to fulfill the principal requirements of clinical efficacy, including several human trial phases [14]. In other words, efficacy trials determine the possibility of an intervention to produce expected results under ideal circumstances or the degree of beneficial effects under real-world clinical settings [15,16].

More importantly, SARS-CoV-2 is the seventh member of the genus $\beta$-coronavirus and the Coronavirdiae family [17]. Genome sequencing of SARS-CoV-2 demonstrated that the virus is almost $79 \%$ and $50 \%$ identical with the previous two SARS-CoV and MERS coronaviruses, respectively [18]. So, it might be very convenient and rational to repurpose the currently available antiviral drugs used against the two previous viral pandemics (SARS and MERS CoV) or have evidence of previous experience. Furthermore, it has been perceived that drug repurposing has already become a "universal strategy" to face the challenges of the COVID-19 pandemic because of several advantages it offers. These include fewer clinical trial steps, the availability of the formulation and distribution of the existing pharmaceutical supply, the possibility of more effective treatment of known combination therapy, the discovery of novel mechanisms of actions of old drugs or new classes of medicines, [19] and the elimination of "activation barriers" in the early stages of research, thus ensuring the rapid advancement of any project to disease-oriented research [20]. 
Several FDA-approved available antiviral drugs, alone or in combination, have been screened clinically for their extended use since the early phase of the current pandemic to find a safe and effective treatment option against COVID-19 [21,22], and many clinical trials of these antiviral drugs are still ongoing. However, an in-depth understanding is required from current clinical literature reports to execute integrated approaches between computational and experimental methods to guarantee high success rates of repositioned drugs. Moreover, multiple challenges associated with repurposed drugs have been identified, including dose adjustments, route of administration, acute/chronic toxicity, appropriate delivery systems, etc. $[23,24]$. Although many preliminary studies exhibited promising results, several extensive clinical investigations reported contradictory findings with significant adverse effects of these elongated applications of antiviral drugs. However, numerous clinical trials conducted with larger samples/patients have recently disclosed many mixed results, which needs careful study. Therefore, it is essential to review comprehensively the uses of repurposed drugs focusing on the therapeutic strategies, advantages, adverse drug reactions, and respective delivery approaches for instigating an instrumental battle against COVID-19. Likewise, it is also necessary to know the clear disease pathology and critical strategies to identify new drugs capable of protecting against highly contagious viral infections, including the SARS-CoV-2 infection [25]. This article summarizes the current understanding of clinical efficacy and the adverse drug reaction of various antiviral drugs used for SARS-CoV-2-infected patients across the world. Here, we also illustrated Figure 1 to represent the chemical structures of the seven repurposed antivirals drugs for use against COVID-19.<smiles>C=CCOC(=O)C1=C[C@H](OC(CC)CC)[C@@H](NC(C)=O)[C@H](N)C1</smiles><smiles>CC(C)CN(C[C@H](O)[C@@H](Cc1ccccc1)NC(=O)O[C@@H]1CCOC1)S(=O)(=O)c1ccc(N)cc1</smiles><smiles>CCOC(=O)c1c(CSc2ccccc2)n(C)c2cc(Br)c(O)c(CN(C)C)c12</smiles>

Figure 1. Chemical structures of the selected most promising antiviral drugs against COVID-19. 


\section{Methods}

\section{Search and Data Collection}

The scientific articles published from January 2020 to June 2021 on several repurposed antiviral drugs that had potentiality against COVID-19 were downloaded from various databases such as Google Scholar, Semantic Scholar, PubMed, Scopus, Europe PMC, ScienceDirect Journals, SpringerLink, Wiley, Taylor \& Francis, and so on, with several preprint servers. We utilized several suitable keywords and used those words individually or in combination to find the data regarding the target topic. The keywords included "COVID19", "coronavirus diseases 2019", "SARS-CoV-2", "clinical trials", "observational study", "cohort study", "safety and efficacy", "remdesivir", "favipiravir", "lopinavir/ritonavir", "interferons", "arbidol", "umifenovir", "oseltamivir", "darunavir", and so on. We included the human studies designed as randomized or non-randomized, open-label, controlled clinical trials, and observational or cohort studies to find a clear understanding of the safety and efficacy of repurposed antiviral drugs against mild, moderate, and severe cases of COVID-19. However, we excluded case reports or case series in this review paper. In addition, the current review rejected the data containing a limited number of subjects, weak statistical analysis, or vague conclusions.

\section{Promising Antiviral Drugs against SARS-CoV-2: Results from Human Studies}

\subsection{Remdesivir ( $R D V)$}

Remdesivir (RDV, GS-5734), a nucleoside monophosphate analog, has emerged as one of the most promising clinically investigated drugs to treat SARS-CoV-2 infection from the beginning phase of the COVID-19 outbreak. RDV was preliminarily developed for treating acute Ebola virus disease [26]. Mechanistically, RDV acts as a broad-spectrum antiviral drug by inhibiting RNA-dependent RNA polymerase (RdRp) enzyme from restricting viral replication, and it has already shown its efficacy against SARS-CoV-1 and MERS-CoV through the improvement of lung infection $[27,28]$.

Grein et al. [29] conducted a cohort study on 61 patients hospitalized with severe COVID-19 who were administered RDV as a compassionate therapy for 10 days (200 $\mathrm{mg}$ on the first day and $100 \mathrm{mg}$ for the next nine days) and observed clinical improvement in $68 \%$ of patients ( $n=36$ out of 53). Antinori et al. [30] conducted another open-label, prospective study in Italy on 35 patients (ICU $=18$ and non-ICU $=17$ ) and observed a more beneficial effect on non-ICU patients after 10 days of therapy of RDV. Although the study recognized some mentionable adverse effects like acute kidney injury and hypertransaminasemia for $22.8 \%$ and $42.8 \%$ of patients, respectively, the study findings recommended a larger randomized controlled clinical trial to evaluate the safety and efficacy of RDV against COVID-19. Pasquini et al. [31] assessed the efficacy of RDV on 51 ICU-admitted patients of an average age of 67 years (IQR $=59.0-75.5)$, of which 25 were treated with the drug. The study reported that the RDV group showed a significantly lower fatality rate (56\% vs. $92 \%$, $p=0.001$ ) and was associated with better survival (OR 3.506; 95\% confidence interval $(\mathrm{CI})=1.768-6.954 ; p<0.001$ ). In another prospective observational study conducted on 48 COVID-19 patients with dialysis-dependent end-stage renal disease, Aiswarya et al. [32] concluded that early treatment with RDV (within $48 \mathrm{~h}$ ) appeared to shorten recovery and discharge time with safety and good tolerability. In a comparative analysis of 1130 patients $(\mathrm{RDV}=312$ and non-RDV $=818)$, Olender et al. [33] reported that the drug exhibited a $15.4 \%$ greater recovery rate than in the non-RDV cohort group and reduced mortality by $4.9 \%$ in severe COVID-19 patients. 
A randomized, placebo-controlled, double-blind clinical trial $(n=1062 ; \mathrm{RDV}=541$ and placebo $=521)$ conducted by Beigel et al. [28] showed that those who took RDV exhibited lessened recovery time (10 days) than those with placebo (15 days), as well as a reduced mortality rate (RDV vs. placebo $=7.1 \%$ vs. $11.9 \%$, respectively). Goldman et al. [34] conducted a phase 3, open-level control trial that displayed the potent antiviral activity of RDV against SARS-CoV-2, with some adverse effects such as nausea (9\%), worsening respiratory failure $(8 \%)$, elevated alanine aminotransferase level $(7 \%)$, and constipation $(7 \%)$. A retrospective comparative study was carried out by Garibaldi et al. [35], which presented the result of a shorter improvement time in the test group than the control group. A large observational study evaluated no statistical difference in efficacy between the RDV and control group [36]. An open-level, randomized clinical trial with RDV initiated by Wang et al. [37] reported no significant clinical benefits and suggested more extensive studies to confirm numerical reduction time for clinical improvement patients in the earlier stage. In another comparative study [38], no statistically significant difference was reported between clinical status and standard care. Another placebo-controlled, double-blinded, randomized trial conducted by Kalil et al. [39] demonstrated the potential activity of the antiviral drugs against COVID-19. Falcao et al. [40] conducted an observational cohort study in Lisbon that compared remdesivir treatment to hydroxychloroquine treatment and their adverse effects. Hydroxychloroquine generated more adverse effects (47.5\%) than RDV. In a real-life setting, the RDV regimen reduced hospitalization in both intubated (1.4 days lass) and non-intubated (3.4 days less) patients, although it was not statistically significant [41]. A study compared the efficacy of remdesivir, convalescent plasma, and a combination of both, which eventually revealed a higher survival rate in patients receiving RDV only [42]. In addition, several more randomized controlled clinical trials are undergoing to ensure the safety and efficacy of RDV against COVID-19. All the above-mentioned study findings and details of randomized characterizations of RDV and other antivirals drugs are tabulated in Table 1. 
Table 1. Summary of the latest clinical trials and observational studies to investigate the safety and efficacy several repurposed antiviral drugs against COVID-19.

\begin{tabular}{|c|c|c|c|c|c|c|c|c|}
\hline Drug & Reference & Study Type & Country & $n$ & ROA & Results & ADR & Interpretation \\
\hline RDV & $\begin{array}{l}\text { Beigel } \\
\text { et al. [28] }\end{array}$ & $\begin{array}{c}\text { RCT } \\
\text { (NCT04280705) }\end{array}$ & $\begin{array}{c}\text { USA, } \\
\text { Denmark, UK, } \\
\text { Greece, Germany, } \\
\text { Korea, Mexico, } \\
\text { Spain, Japan, and Singapore }\end{array}$ & $\begin{array}{c}1059 \text { patients } \\
(\mathrm{T}=541 \text { and } \mathrm{C}=521)\end{array}$ & IV & $\begin{array}{l}\text { RDV showed shortened } \\
\text { recovery time (from } 15 \text { to } 11 \\
\text { days) and reduced mortality } \\
\text { rate by } 4.8 \%\end{array}$ & $\begin{array}{c}\text { Serious ADR: } \mathrm{T} \text { vs. } \mathrm{C}= \\
24.6 \% \text { vs. } 31.6 \%\end{array}$ & Warrants RCT \\
\hline RDV & $\begin{array}{l}\text { Grein } \\
\text { et al. [29] }\end{array}$ & Cohort & $\begin{array}{l}\text { USA, Europe, Canada, } \\
\text { and Japan }\end{array}$ & 61 & IV & $\begin{array}{l}68 \% \text { patients }(n=36 \text { out of } 53 \text { ) } \\
\text { showed clinical improvement }\end{array}$ & $\begin{array}{c}\text { Multiple organ } \\
\text { dysfunction syndrome, } \\
\text { septic shock, acute } \\
\text { kidney injury, and } \\
\text { hypotension }\end{array}$ & Warrants RCT \\
\hline RDV & $\begin{array}{l}\text { Antinori } \\
\text { et al. [30] }\end{array}$ & $\begin{array}{c}\text { Open label } \\
\text { (Observational) }\end{array}$ & Italy & $\begin{array}{l}35 \text { patients }(\mathrm{ICU}=18 \\
\text { and Non-ICU }=17)\end{array}$ & IV & $\begin{array}{l}\text { Observed more beneficial } \\
\text { effect for non-ICU patients } \\
\text { after } 10 \text { days of therapy }\end{array}$ & $\begin{array}{l}\text { Hyper-transaminasemia } \\
\text { and acute kidney injury }\end{array}$ & Warrants RCT \\
\hline RDV & $\begin{array}{l}\text { Pasquini } \\
\text { et al. [31] }\end{array}$ & Retrospective & Italy & $\begin{array}{c}51 \text { patients } \\
(\mathrm{T}=25, \mathrm{C}=26)\end{array}$ & IV & $\begin{array}{l}\text { Significantly reduced fatality } \\
\text { rate }(56 \% \text { vs. } 92 \% ; p<0.001) \\
\text { and improved survival rate } \\
(\mathrm{OR}=3.506,95 \% \mathrm{CI}=1.768 \\
\text { to } 6.954 ; p<0.001)\end{array}$ & NA & $\begin{array}{l}\text { Significant survaival } \\
\text { might be associated } \\
\text { with RDV use }\end{array}$ \\
\hline RDV & $\begin{array}{l}\text { Aiswarya } \\
\text { et al. [32] }\end{array}$ & Cohort & India & 48 & IV & $\begin{array}{l}\text { Early treatment of RDV } \\
\text { (within } 48 \mathrm{hrs} \text { ) reduced } \\
\text { recovery and discharge time } \\
\text { with safety and good } \\
\text { tolerability. }\end{array}$ & $\begin{array}{l}\text { Acute kidney injury and } \\
\text { hypotension }\end{array}$ & Warrants RCT \\
\hline RDV & $\begin{array}{l}\text { Olender } \\
\text { et al. [33] }\end{array}$ & $\begin{array}{c}\text { Open level } \\
\text { (NCT04292899) }\end{array}$ & $\begin{array}{l}\text { USA, Italy, Spain, Germany, } \\
\text { Hong Kong, Singapore, S. } \\
\text { Korea, and Taiwan }\end{array}$ & $\begin{array}{c}1130 \text { patients } \\
(\mathrm{T}=312 \text { and } \mathrm{C}=818)\end{array}$ & IV & $\begin{array}{c}\text { RDV exerted } 15.4 \% \text { more } \\
\text { recovery (adjusted odds ratio } \\
(\mathrm{aOR}=2.03 ; 95 \% \mathrm{CI}= \\
1.34-3.08, p=0.001) \text { and } \\
\text { reduced mortality of } 4.9 \% \\
(\mathrm{aOR}=0.38 ; 95 \% \mathrm{CI}= \\
0.22-0.68, p=0.001) \text { in severe } \\
\text { COVID-19 patients. }\end{array}$ & NA & Warrants RCT \\
\hline
\end{tabular}


Table 1. Cont.

\begin{tabular}{|c|c|c|c|c|c|c|c|c|}
\hline Drug & Reference & Study Type & Country & $n$ & ROA & Results & ADR & Interpretation \\
\hline RDV & $\begin{array}{l}\text { Goldman } \\
\text { et al. [34] }\end{array}$ & RCT (NCT04292899) & $\begin{array}{c}\text { USA, Italy, } \\
\text { Spain, Germany, Hong Kong, } \\
\text { Singapore, South Korea } \\
\text { and Taiwan }\end{array}$ & $\begin{array}{l}397 \text { patients (10-day } \\
\text { course for } 200 \text { and } \\
\text { 5-day course for 197) }\end{array}$ & IV & $\begin{array}{l}\text { No significant difference } \\
\text { between 5- or 10-day course }\end{array}$ & $\begin{array}{c}\text { Nausea }(9 \%), \\
\text { worsening respiratory } \\
\text { failure }(8 \%), \text { elevated } \\
\text { alanine aminotransferase } \\
\text { level }(7 \%), \text { and } \\
\text { constipation }(7 \%) .\end{array}$ & Warrants RCT \\
\hline RDV & $\begin{array}{l}\text { Garibaldi } \\
\text { et al. [35] }\end{array}$ & Retrospective & USA & $\begin{array}{l}2309 \text { patients } \\
(\mathrm{RDV}=158, \mathrm{RDV}+ \\
\begin{aligned} \mathrm{CTS} & =184 \text { and } \\
\mathrm{C} & =1957)\end{aligned}\end{array}$ & NA & $\begin{array}{l}\text { Clinical improvement time is } \\
\text { shorter in RDV group } \\
\text { compared to control group. }\end{array}$ & $\begin{array}{l}\text { Increased levels of liver } \\
\text { enzyme or bilirubin } \\
(n=4), \text { kidney failure of } \\
\text { unclear cause }(n=2), \\
\text { nausea }(n=1) \text {, epistaxis } \\
\text { and tachycardia }(n=1), \\
\text { neck and mouth itching } \\
(n=1)\end{array}$ & $\begin{array}{l}\text { Warrants further } \\
\text { study }\end{array}$ \\
\hline RDV & $\begin{array}{l}\text { Tsuzuki } \\
\text { et al. [36] }\end{array}$ & Observational study & Japan & $\begin{array}{c}269 \text { patients }(T=74 \\
\text { and } C=195)\end{array}$ & NA & $\begin{array}{l}\text { No statistical significance } \\
\text { was observed in fatality rate } \\
\text { or length of hospital stay }\end{array}$ & $\begin{array}{c}\text { Elevation of liver } \\
\text { enzyme and rash }(n=2)\end{array}$ & $\begin{array}{l}\text { Warrants further } \\
\text { study }\end{array}$ \\
\hline RDV & $\begin{array}{l}\text { Wang } \\
\text { et al. [37] }\end{array}$ & RCT (NCT04257656) & China & $\begin{array}{l}237 \text { patients }(\mathrm{T}=158 \\
\text { and } \mathrm{C}=79)\end{array}$ & IV & $\begin{array}{l}\text { No significant time difference } \\
\text { for clinical improvement } \\
\text { between the two groups } \\
(\mathrm{HR}=1.23,95 \% \\
\mathrm{CI}=0.87-1.75)\end{array}$ & $\begin{array}{c}\text { Adverse effects were } \\
\text { reported for both groups; } \\
\mathrm{T}=102(66 \%) \text { and } \mathrm{C}=50 \\
(64 \%)\end{array}$ & $\begin{array}{c}\text { Numerical time } \\
\text { reduction to clinical } \\
\text { benefit was reported } \\
\text { that warrants larger } \\
\text { study }\end{array}$ \\
\hline $\mathrm{RDV}$ & $\begin{array}{l}\text { Spinner } \\
\text { et al. [38] }\end{array}$ & $\begin{array}{c}\text { RCT } \\
\text { (NCT04292730) }\end{array}$ & $\begin{array}{l}\text { USA, } \\
\text { Europe, and Asia }\end{array}$ & $\begin{array}{l}596 \text { patients (T: } \\
\text { 10-day course for } \\
\text { 197, 5-day course for } \\
\text { 199, and C: } 200 \text { ) }\end{array}$ & IV & $\begin{array}{c}\text { No statistically significant } \\
\text { change between T and C } \\
(p=0.18 \text { by Wilcoxon rank } \\
\text { sum test) }\end{array}$ & $\begin{array}{l}\text { Nausea ( } 10 \% \text { vs. } 3 \%) \\
\text { hypokalemia }(6 \% \text { vs. } \\
2 \%), \text { and headache } \\
(5 \% \text { vs. } 3 \%)\end{array}$ & RCT is warranted \\
\hline $\mathrm{RDV}+\mathrm{BCN}$ & Kalil et al. [39] & RCT (NCT04401579) & $\begin{array}{l}\text { USA, UK, Singapore, } \\
\text { South Korea, Mexico, Japan, } \\
\text { Spain, and Denmark }\end{array}$ & $\begin{array}{c}1033 \text { patients } \\
(\mathrm{T}=515 \text { and } \mathrm{C}=518)\end{array}$ & IV, oral & $\begin{array}{c}\text { At } 28 \text {-day fatality rate: } \mathrm{T}= \\
5.1 \% \text { and } \mathrm{C}=7.8 \% \text { (HR for } \\
\text { death }=0.65 ; 95 \% \mathrm{CI}, 0.39 \\
\text { to } 1.09) .\end{array}$ & $\begin{array}{l}\text { Some common ADRs } \\
\text { like hyperglycemia, } \\
\text { anemia, decreased } \\
\text { lymphocyte count, and } \\
\text { acute kidney injury were } \\
\text { present in both groups }\end{array}$ & $\begin{array}{l}\text { The combination } \\
\text { therapy was superior } \\
\text { to only RDV. }\end{array}$ \\
\hline RDV & $\begin{array}{l}\text { Falcao } \\
\text { et al. [40] }\end{array}$ & $\begin{array}{l}\text { Observational } \\
\text { cohort study }\end{array}$ & Lisbon, Portugal & $\begin{array}{c}149 \text { patients } \\
(\mathrm{HCQ}=101 \text { and } \\
\mathrm{RDV}=48)\end{array}$ & Oral & $\begin{array}{c}\text { ADRs were more significant } \\
\text { the in case of HCQ }(47.5 \%) \\
\text { than RDV }(12.5 \%) .\end{array}$ & $\begin{array}{l}\text { RDV group developed } \\
\text { ADRs like hepatobiliary } \\
\text { disorder }(8.3 \%), \text { acute } \\
\text { renal failure, nervous } \\
\text { system disorder, and } \\
\text { others }(2.1 \%) .\end{array}$ & $\begin{array}{l}\text { Warrants larger } \\
\text { sample size and } \\
\text { follow-up study }\end{array}$ \\
\hline
\end{tabular}


Table 1. Cont.

\begin{tabular}{|c|c|c|c|c|c|c|c|c|}
\hline Drug & Reference & Study Type & Country & $n$ & ROA & Results & ADR & Interpretation \\
\hline $\mathrm{RDV}$ & $\begin{array}{l}\text { Goldberg } \\
\text { et al. [41] }\end{array}$ & $\begin{array}{c}\text { Real-life } \\
\text { observational }\end{array}$ & Israel & $\begin{array}{l}142 \text { patients }(T=29 \\
\text { and } C=113)\end{array}$ & IV & $\begin{array}{c}\text { Reduced hospitalization in } \\
\text { case of both non-intubated } \\
\text { and intubated patients ( } 3.1 \\
\text { vs. } 1.4 \text { days, respectively; } \\
p>0.05)\end{array}$ & NA & $\begin{array}{l}\text { Warrants further } \\
\text { study as sample size } \\
\text { was small }\end{array}$ \\
\hline $\begin{array}{l}\mathrm{RDV} / \mathrm{CP} / \mathrm{RDV}+ \\
\mathrm{CP}\end{array}$ & $\begin{array}{l}\text { Padilla } \\
\text { et al. [42] }\end{array}$ & $\begin{array}{c}\text { Retrospective } \\
\text { observational study }\end{array}$ & California, USA & $\begin{array}{c}106 \text { patients }(\mathrm{RDV}= \\
11, \mathrm{CP}=53, \text { and } \\
\mathrm{RDV}+\mathrm{CP}=42)\end{array}$ & IV & $\begin{array}{l}\text { Survival rate was higher with } \\
\text { RDV alone than with } \\
\text { combination therapy or } \\
\text { CP alone. }\end{array}$ & NA & $\begin{array}{l}\text { Warrants further } \\
\text { study }\end{array}$ \\
\hline FPV & Chen et al. [44] & $\begin{array}{c}\text { RCT } \\
\text { (ChiCTR2000030254). }\end{array}$ & China & $\begin{array}{c}240 \text { patients }(\mathrm{T}=120, \\
\mathrm{C}=120)\end{array}$ & NA & $\begin{array}{l}\text { T showed shorter latency } \\
\text { recovery from fever } \\
\text { (difference: } 1.70 \text { days, } \\
p<0.0001 \text { ) and cough } \\
\text { (difference: } 1.75 \text { days, } \\
p<0.0001 \text { ) }\end{array}$ & $\begin{array}{l}\text { Increased serum uric } \\
\text { acid } 16(13.79 \%) \text { in } \\
\text { FPVipiravir group, } \\
\quad p=0.0014\end{array}$ & $\begin{array}{l}\text { Adverse effects are } \\
\text { mild and } \\
\text { manageable. }\end{array}$ \\
\hline FPV & $\begin{array}{l}\text { The New Indian } \\
\text { Express [46] }\end{array}$ & $\mathrm{RCT}$ & India & $\begin{array}{c}150(\mathrm{FPV}=75 \text { and } \\
\quad \text { control }=75)\end{array}$ & Oral & $\begin{array}{c}\text { Primarily FPV showed } 28.6 \% \\
\text { numerical faster viral } \\
\text { clearance }\end{array}$ & NA & Warrants RCT \\
\hline FPV & $\begin{array}{l}\text { The Daily } \\
\text { Star [47] }\end{array}$ & $\mathrm{RCT}$ & Bangladesh & 50 & Oral & $\begin{array}{l}\text { FPV showed } 44 \% \text { more viral } \\
\text { clearance than placebo }\end{array}$ & $\begin{array}{c}\text { No significant side effect } \\
\text { was reported. }\end{array}$ & Warrants RCT \\
\hline FPV & Ucan et al. [48] & Retrospective cohort & Turkey & $\begin{array}{c}144 \\
(\mathrm{FPV}+\mathrm{HCQ} \\
(\text { early })=48, \mathrm{FPV}+ \\
\mathrm{HQ} \text { (late) }=48 \text { and } \\
\mathrm{HQ}=48)\end{array}$ & Oral & $\begin{array}{l}\text { Early starting of FPV had an } \\
\text { impact on PCR negativity } \\
\text { and the progression of } \\
\text { the disease. }\end{array}$ & $\begin{array}{l}\text { Diarrhea, nausea, } \\
\text { and vomiting }\end{array}$ & Needs RCT \\
\hline
\end{tabular}


Table 1. Cont.

\begin{tabular}{|c|c|c|c|c|c|c|c|c|}
\hline Drug & Reference & Study Type & Country & $n$ & ROA & Results & ADR & Interpretation \\
\hline FPV & $\begin{array}{l}\text { Alamer } \\
\text { et al. [49] }\end{array}$ & Retrospective & Saudi Arabia & $\begin{array}{c}457(\mathrm{FPV}=234 \text { and } \\
\mathrm{C}=223)\end{array}$ & Oral & $\begin{array}{l}\text { Improvement of discharge } \\
\text { rate and less progression to } \\
\text { ventilation; no effect on } \\
\text { mortality in severe cases }\end{array}$ & $\begin{array}{c}\text { Acute kidney injury, } \\
\text { increased ALT, AST, } \\
\text { bilirubin, cardiovascular } \\
\text { effects, constipation, } \\
\text { seizure, hypercalcemia, } \\
\text { hyperphosphatemia, and } \\
\text { hypermagnesemia }\end{array}$ & Warrants RCT \\
\hline FPV & Zhao et al. [50] & $\mathrm{RCT}$ & China & $\begin{array}{c}55(\mathrm{FPV}=36 \text { and } \\
\mathrm{C}=19)\end{array}$ & Oral & $\begin{array}{l}\text { Improvement in virus } \\
\text { shedding and CRP } \\
\text { decreasing }\end{array}$ & $\begin{array}{l}\text { Elevated AST and ALT, } \\
\text { hyperuricemia, } \\
\text { hypernatremia, diarrhea, } \\
\text { and nausea. }\end{array}$ & Warrants RCT \\
\hline FPV & $\begin{array}{l}\text { Udwadia } \\
\text { et al. [51] }\end{array}$ & $\mathrm{RCT}$ & India & $\begin{array}{c}150 \text { patients }(T=75 \\
\text { and } C=75)\end{array}$ & Oral & $\begin{array}{l}\text { Median required time of } \\
\text { reducing viral load between } \\
\text { T and C was } 5 \text { days vs. } \\
7 \text { days and clinically median } \\
\text { cure time was } 3 \text { days vs. } \\
5 \text { days, respectively }\end{array}$ & $\begin{array}{l}\text { ADR: T vs. } C=36 \% \text { vs. } \\
8 \% \text {, respectively }\end{array}$ & $\begin{array}{l}\text { T showed significant } \\
\text { improvement in time } \\
\text { to clinical recovery }\end{array}$ \\
\hline $\operatorname{rhIFN}-\alpha$ & $\begin{array}{l}\text { Meng } \\
\text { et al. [52] }\end{array}$ & RTC (NCT04320238) & China & $\begin{array}{l}2944 \text { patients (low } \\
\text { risk }=2415 \text { and high } \\
\text { risk }=529)\end{array}$ & $\begin{array}{l}\text { Nasal } \\
\text { drop }\end{array}$ & $\begin{array}{l}\text { Observed negative clinical } \\
\text { symptoms for pneumonia in } \\
\text { both high- and low-risk } \\
\text { groups after } 28 \text { days }\end{array}$ & $\begin{array}{l}\text { Flu-like symptoms } \\
\text { (burning pain and } \\
\text { itching), allergic } \\
\text { reactions (rash, nausea, } \\
\text { chest distress, } \\
\text { palpitation, and flushing) }\end{array}$ & Warrants RCT \\
\hline IFN-alpha $2 b$ & $\begin{array}{l}\text { Pandit } \\
\text { et al. [53] }\end{array}$ & $\mathrm{RCT}$ & India & $\begin{aligned} 40(\mathrm{~T} & =20 \text { and } \\
\mathrm{C} & =20)\end{aligned}$ & SC & $\begin{array}{c}\text { Better clinical improvement } \\
(\mathrm{IFN}=95 \% \text { and } \mathrm{C}=68.42 \%) \\
\text { and viral shedding } \\
\text { (IFN }=95 \% \text { and } \mathrm{C}=68 \%)\end{array}$ & $\begin{array}{l}\text { Respiratory distress, } \\
\text { hypoxia, nausea, } \\
\text { vomiting, mouth } \\
\text { dryness, and headache }\end{array}$ & $\begin{array}{l}\text { Further confirmatory } \\
\text { studies needed }\end{array}$ \\
\hline IFN-alpha $2 b$ & Yu et al. [54] & Retrospective & China & $\begin{array}{c}1401(\mathrm{~T}=852 \text { and } \\
\mathrm{C}=549)\end{array}$ & Inhalation & $\begin{array}{l}\text { Lower viral shedding time, } \\
\text { improved clinical outcome }\end{array}$ & NA & Warrants RCT \\
\hline IFN-lambda & Feld et al. [55] & $\mathrm{RCT}$ & Canada & $\begin{array}{c}60(\text { IFN }=30 \text { and } \\
\quad \mathrm{C}=30)\end{array}$ & SC & $\begin{array}{l}\text { Shortening of viral shedding } \\
\text { duration, prevention of } \\
\text { clinical deterioration, and } \\
\text { acceleration of viral decline. }\end{array}$ & $\begin{array}{l}\text { Confusion, rectal } \\
\text { bleeding, pneumonia, } \\
\text { and pulmonary } \\
\text { embolism }\end{array}$ & $\begin{array}{l}\text { Warrants larger } \\
\text { studies }\end{array}$ \\
\hline ARB & $\begin{array}{l}\text { Wang } \\
\text { et al. [56] }\end{array}$ & Cohort & China & 69 & NA & $\begin{array}{c}\text { Improved hospital discharge } \\
\text { rate and reduced mortality } \\
\text { rate by } 7.5 \%\end{array}$ & NA & $\begin{array}{l}\text { Study with larger } \\
\text { samples needed }\end{array}$ \\
\hline
\end{tabular}


Table 1. Cont

\begin{tabular}{|c|c|c|c|c|c|c|c|c|}
\hline Drug & Reference & Study Type & Country & $n$ & ROA & Results & ADR & Interpretation \\
\hline $\mathrm{ARB}$ & Lian et al. [57] & Retrospective & China & $\begin{array}{l}81 \text { patients }(\mathrm{ARB}= \\
45 \text { and control }=36)\end{array}$ & NA & $\begin{array}{l}\text { ARB showed better CT scores } \\
\text { than control (IQR 7e14) vs. } 8 \\
\text { (IQR 5e10), } p<0.05\end{array}$ & $\begin{array}{c}5 / 45(11 \%) \text { ARB group } \\
\text { and } 3 / 36(8 \%) \text { control } \\
\text { group showed digestive } \\
\text { symptoms such as } \\
\text { diarrhea and nausea } \\
(p=0.49)\end{array}$ & Warrants RCT \\
\hline ARB & Zhu et al. [58] & Retrospective & China & $\begin{array}{c}50 \text { patients } \\
(\mathrm{ARB}=16 \text { and } \\
\mathrm{LPV} / \mathrm{RTV}=34)\end{array}$ & NA & $\begin{array}{l}\text { After day } 14 \text {, viral load was } \\
\text { found between (ARB vs. } \\
\text { LPV } / \text { RTV }=0 \% \text { vs. } 44.1 \% \text { ) }\end{array}$ & NA & $\begin{array}{l}\text { ARB superior to } \\
\text { LPV/RTV }\end{array}$ \\
\hline $\mathrm{ARB}$ & Chen et al. [59] & Observational & China & $\begin{array}{c}62 \text { patients }(T=42 \\
\text { and } C=20)\end{array}$ & Oral & $\begin{array}{l}\text { Test group reduced hospital } \\
\text { stay duration more than } \\
\text { control group }\end{array}$ & $\begin{array}{c}\text { Nausea (test: } n=7, \\
\text { control: } n=3 \text { ), diarrhea } \\
\text { (test: } n=2 \text {, control: } \\
n=1 \text { ), and dizziness } \\
\text { (test: } n=2 \text {, control: } n=1 \text { ) }\end{array}$ & $\begin{array}{l}\text { Warrants further } \\
\text { study }\end{array}$ \\
\hline ARB, oseltamivir & Liu et al. [60] & Retrospective cohort & China & 504 & NA & $\begin{array}{l}\text { Both drugs reduced } \\
\text { fatality rate }\end{array}$ & Nausea & $\begin{array}{l}\mathrm{ARB} \text { and oseltamivir } \\
\text { reduced fatality rate. }\end{array}$ \\
\hline ARB & Yang et al. [61] & $\mathrm{RCT}$ & China & $\begin{array}{c}164 \text { patients } \\
(\mathrm{ARB}=82 \text { and } \\
\text { non- } \mathrm{ARB}=82)\end{array}$ & Oral & $\begin{array}{l}\text { In the ARB group } \\
\text { The uninfected rate was } \\
\text { significantly higher than in } \\
\text { the non-ARB group. }\end{array}$ & NA & $\begin{array}{l}\text { Needs a multicenter } \\
\text { cohort study }\end{array}$ \\
\hline $\mathrm{ARB}$ & Zeng et al. [62] & Retrospective cohort & China & $\begin{array}{c}1019(\mathrm{ARB}=788 \text { and } \\
\mathrm{C}=231)\end{array}$ & NA & $\begin{array}{c}\text { ARB-treatment results in less } \\
\text { in-hospital death for patients } \\
\text { with severe and critical } \\
\text { COVID-19. }\end{array}$ & NA & Warrants RCT \\
\hline Oseltamivir & Tan et al. [63] & Cohort & China & $\begin{array}{c}333 \text { patients } \\
\text { (oseltamivir }=14, \\
\text { ARB }=277, \\
\text { corticosteroid }=15, \\
\text { HCQ }=8, \\
\text { LPV } / \text { RTV }=14 \text { ) }\end{array}$ & NA & $\begin{array}{c}\text { The oseltamivir group } \\
\text { showed a significantly } \\
\text { shorter hospital stay duration } \\
\text { than the ARB, corticosteroids, } \\
\text { and } \\
\text { lopinavir/ritonavir groups. }\end{array}$ & NA & $\begin{array}{c}\text { Oseltamivir } \\
\text { prudently } \\
\text { considered as a } \\
\text { combination therapy }\end{array}$ \\
\hline Oseltamivir & $\begin{array}{l}\text { Moreno } \\
\text { et al. [64] }\end{array}$ & Observational & Spain & $\begin{array}{l}2124(\mathrm{~T}(\text { early })=529 \\
\text { and C (late })=1595)\end{array}$ & NA & $\begin{array}{l}\text { Early treatment was } \\
\text { associated with reduced } \\
\text { mortality of critically ill } \\
\text { patients infected with } \\
\text { influenza pneumonia. }\end{array}$ & NA & $\begin{array}{l}\text { Further studies } \\
\text { needed }\end{array}$ \\
\hline
\end{tabular}


Table 1. Cont.

\begin{tabular}{|c|c|c|c|c|c|c|c|c|}
\hline Drug & Reference & Study Type & Country & $n$ & ROA & Results & ADR & Interpretation \\
\hline $\mathrm{DRV} / \mathrm{c}$ & Deng et al. [65] & Retrospective & China & $\begin{array}{l}66 \text { patients }(\mathrm{DRV} / \mathrm{c}= \\
32 \text { and control }=34)\end{array}$ & Oral & $\begin{array}{c}\mathrm{DRV} / \mathrm{c} \text { significantly } \\
\text { shortened nucleic acid } \\
\text { conversion duration from the } \\
\text { onset of symptoms } \\
\text { to admission. }\end{array}$ & $\begin{array}{l}\text { Respiratory failure, } \\
\text { upset stomach }\end{array}$ & $\begin{array}{l}\text { Further studies } \\
\text { needed }\end{array}$ \\
\hline $\mathrm{DRV} / \mathrm{c}$ & Kim et al. [66] & Retrospective & South Korea & $\begin{array}{l}110 \text { patients }(\mathrm{DRV}-\mathrm{c} \\
\text { group }=14 \text { and } \\
\text { control }=96)\end{array}$ & NA & $\begin{array}{c}\text { Overall, the DRV/c group } \\
\text { showed a lower fatality rate } \\
\text { than the control group (odds } \\
\text { ratio (OR) } 0.20,95 \% \mathrm{CI}= \\
0.04-0.89, p=0.035 \text { ). }\end{array}$ & NA & $\begin{array}{c}\text { DRV-c showed } \\
\text { significant survival } \\
\text { benefit in critically ill } \\
\text { Patients. }\end{array}$ \\
\hline $\mathrm{DRV} / \mathrm{c}$ & Chen et al. [67] & RCT (NCT04252274) & China & $\begin{array}{l}30 \text { patients }(\mathrm{DRV} / \mathrm{c}= \\
15 \text { and control }=15)\end{array}$ & Oral & $\begin{array}{l}\text { The difference in negative } \\
\text { PCR conversion rate in } \\
\text { between two groups at day } 7 \\
\text { was DRV / c vs. control = } \\
9 / 15(60.0 \%) \text { vs. } 7 / 15 \\
(46.7 \%), \text { respectively, } p=0.72 .\end{array}$ & $\begin{array}{c}\text { In the DRV / c group, } \\
\text { diarrhea }(20 \%) \text {, anemia, } \\
\text { elevated transaminase } \\
\text { levels }(13.3 \%) \text {, and renal } \\
\text { dysfunction (13.3\%) were } \\
\text { observed. }\end{array}$ & $\begin{array}{l}5 \text { days of DRV /c did } \\
\text { not increase the rate } \\
\text { of negative } \\
\text { conversion vs. } \\
\text { standard of } \\
\text { care alone. }\end{array}$ \\
\hline $\mathrm{FPV}+\mathrm{HQ}$ & $\begin{array}{l}\text { Guner } \\
\text { et al. [68] }\end{array}$ & Retrospective & Turkey & $\begin{array}{c}824(\mathrm{HQ}=604 \\
\mathrm{FPV}=100 \text { and } \mathrm{FPV}+ \\
\mathrm{HQ}=120)\end{array}$ & Oral & $\begin{array}{l}\text { No statistically significant } \\
\text { difference between the } \\
\text { three groups }\end{array}$ & NA & Warrants RCT \\
\hline $\begin{array}{l}\text { FPV vs. } \\
\text { LPV/RTV }\end{array}$ & $\begin{array}{l}\text { Kocayiğit } \\
\text { et al. [70] }\end{array}$ & Observational & Turkey & $\begin{array}{c}107(\mathrm{FPV}=65 \text { and } \\
\mathrm{RTV} / \mathrm{LPV}=42)\end{array}$ & Oral & $\begin{array}{l}\text { Significantly shorter hospital } \\
\text { stay in the case of FPV }\end{array}$ & $\begin{array}{l}\text { Coinfection, ARDS, acute } \\
\text { kidney disease, } \\
\text { and MODS }\end{array}$ & $\begin{array}{l}\text { Warrants larger } \\
\text { sample }\end{array}$ \\
\hline FPV vs. HCQ & $\begin{array}{l}\text { Dabbous } \\
\text { et al. [71] }\end{array}$ & RCT & Egypt & $\begin{aligned} 100(\mathrm{FPV} & =50 \text { and } \\
\mathrm{HCQ} & =50)\end{aligned}$ & Oral & $\begin{array}{c}\text { No significant difference in } \\
\text { hospital stay }\end{array}$ & $\begin{array}{c}\text { Elevated D-dimer and } \\
\text { ferritin, cardiovascular } \\
\text { complications }\end{array}$ & $\begin{array}{l}\text { Warrants larger } \\
\text { sample }\end{array}$ \\
\hline $\begin{array}{l}\text { FPV + IFN } \\
\text { beta-1b }\end{array}$ & $\begin{array}{l}\text { Khamis } \\
\text { et al. [72] }\end{array}$ & RCT (NCT04385095). & Oman & $\begin{array}{c}89 \text { patients }(\mathrm{FPV}+ \\
\text { IFN beta- } 1 \mathrm{~b}=44 \text { and } \\
\text { HCQ }=45)\end{array}$ & Oral & $\begin{array}{l}\text { No significant difference in } \\
\text { hospital stay between FPV } \\
\text { and HCQ (7 vs. } 7 \text { days; } p= \\
0.948), \text { ICU transfer rate } \\
(18.2 \% \text { vs. } 17.8 \% ; p=0.960) \\
\text { or discharge rate }(65.9 \% \text { vs. } \\
68.9 \% ; p=0.764), \text { and fatality } \\
\text { rate was } 11.4 \% \text { vs. } 13.3 \% \text {, } \\
\text { respectively; } p=0.778\end{array}$ & liver injury & $\begin{array}{l}\text { No notable } \\
\text { difference was found } \\
\text { in clinical results } \\
\text { between FPV }+ \\
\text { inhaled IFN beta-1b } \\
\text { and HCQ. }\end{array}$ \\
\hline
\end{tabular}


Table 1. Cont.

\begin{tabular}{|c|c|c|c|c|c|c|c|c|}
\hline Drug & Reference & Study Type & Country & $n$ & ROA & Results & ADR & Interpretation \\
\hline LPV/RTV & Kim et al. [73] & Cohort & Korea & $\begin{array}{c}65 \text { patients } \\
(\mathrm{T}=31, \mathrm{C}=34)\end{array}$ & NA & $\begin{array}{l}\text { No significant difference in } \\
\text { time to clinical development } \\
\text { between } T \text { and } C \text { groups ( } 18 \\
\text { days vs. } 21 \text { days) }\end{array}$ & $\begin{array}{l}\text { One serious ADR was } \\
\text { detected for the T group } \\
\text { and two serious ADRs } \\
\text { were detected for the } \\
\text { C group. }\end{array}$ & Warrants RCT \\
\hline LPV/RTV, HCQ & Lee et al. [74] & Cohort & South Korea & $\begin{array}{c}72 \text { patients } \\
(\mathrm{LPV} / \mathrm{RTV}=45 \text { and } \\
\mathrm{HCQ}=27)\end{array}$ & Oral & $\begin{array}{l}\text { The LPV / RTV group showed } \\
\text { lower failure rate ( } 41 \% \text { vs. } \\
2 \% ; p=0.001 \text { ) and disease } \\
\text { progression than the HCQ } \\
\text { group ( } 18 \% \text { vs. } 44 \%, \\
\text { respectively; } p=0.03 \text { ). }\end{array}$ & $\begin{array}{l}\text { Diarrhea, abnormal } \\
\text { stools, abdominal pain, } \\
\text { nausea, vomiting, and } \\
\text { asthenia }\end{array}$ & $\begin{array}{l}\text { LPV / RTV showed } \\
\text { more efficacy than } \\
\text { HCQ in improving } \\
\text { clinical symptoms }\end{array}$ \\
\hline LPV/RTV & Yu et al. [75] & Cohort & China & 128 & NA & $\begin{array}{l}\text { The significant median } \\
\text { period of viral load reduction } \\
\text { time between with influenza } \\
\text { and without influenza was } \\
17.0 \text { vs. } 12.0 \text { days, } \\
\text { respectively; } p<0.001 \text {. } \\
\text { Besides, the T group showed } \\
\text { faster pneumonia resolution } \\
\text { than the C group ( } 37 \% \text { vs. } 1 \% \text {; } \\
\qquad p=0.001) \text {. }\end{array}$ & NA & $\begin{array}{l}\text { Additional robust } \\
\text { scientific studies } \\
\text { with proper controls } \\
\text { are needed. }\end{array}$ \\
\hline $\begin{array}{c}\mathrm{LPV} / \mathrm{RTV}+ \\
\mathrm{SOC}\end{array}$ & $\begin{array}{l}\text { Lecronier } \\
\text { et al. [76] }\end{array}$ & Retrospective & Paris, France & $\begin{array}{c}80 \text { patients } \\
(\mathrm{LPV} / \mathrm{RTV}+\mathrm{SOC}= \\
20, \mathrm{SOC}=22, \text { and } \\
\mathrm{HCQ}+\mathrm{SCQ}= \\
38 \text { patients })\end{array}$ & Oral & $\begin{array}{l}\text { No significant difference at } \\
\text { ventilator-free days at } \\
28 \text { days and mortality rate (at } \\
\text { 14- and } 28 \text {-days) in these } \\
3 \text { groups }\end{array}$ & NA & Warrants RCT \\
\hline $\begin{array}{c}\mathrm{LPV} / \mathrm{RTV}+ \\
\mathrm{ARB}\end{array}$ & Lan et al. [77] & Retrospective & China & $\begin{array}{c}73 \text { patients }(\mathrm{T}: \\
\mathrm{LPV} / \mathrm{RTV}+\mathrm{ARB}= \\
39 \text { and C: } \\
\mathrm{LPV} / \mathrm{RTV}=34)\end{array}$ & Oral & $\begin{array}{c}\text { The T group showed a } 4.8 \% \\
\text { higher recovery rate and a } \\
\text { 1.5-day shorter hospital stay } \\
\text { than the } C \text { group. }\end{array}$ & NA & $\begin{array}{l}\text { No benefit was } \\
\text { observed between } \\
\text { the two groups. }\end{array}$ \\
\hline $\mathrm{LPV} / \mathrm{RTV}+\mathrm{CQ}$ & Gao et al. [78] & Retrospective & China & $\begin{array}{c}129 \text { patients } \\
(\mathrm{LPV} / \mathrm{RTV}=51 \\
\text { SOC }=59, \text { and } \\
\text { chloroquine }=19)\end{array}$ & Oral & $\begin{array}{l}\text { Neither LPV/RTV nor CQ } \\
\text { improved prognosis or } \\
\text { shortened the clinical course. }\end{array}$ & NA & Warrants RCT \\
\hline
\end{tabular}


Table 1. Cont

\begin{tabular}{|c|c|c|c|c|c|c|c|c|}
\hline Drug & Reference & Study Type & Country & $n$ & ROA & Results & ADR & Interpretation \\
\hline $\mathrm{LPV} / \mathrm{RTV}$ & $\begin{array}{l}\text { Karolyi } \\
\text { et al. [79] }\end{array}$ & Cohort & Austria & $\begin{array}{c}156 \text { patients } \\
(\mathrm{T}=47 \text { and } \mathrm{C}=20)\end{array}$ & IV & $\begin{array}{c}\text { No significant difference in } \\
\text { mortality rate }(8.5 \% \text { vs. } 15 \% \text {; } \\
p=0.418), \text { ICU admission } \\
\text { rate }(12.8 \% \text { vs. } 20 \% ; p=0.47), \\
\text { or hospital stay }(11 \text { days vs. } 9 \\
\text { days, respectively; } p=0.34) \\
\text { was observed. }\end{array}$ & $\begin{array}{l}\text { Diarrhea }(n=7) \text { and liver } \\
\text { enzyme elevation }(n=7)\end{array}$ & Warrants RCT \\
\hline $\mathrm{LPV} / \mathrm{RTV}$ & Shi et al. [80] & $\begin{array}{c}\text { Non-RCT } \\
\text { (ChiCTR2000029400/ } \\
\text { ChiM- } \\
\text { CTR2000002940). }\end{array}$ & China & $\begin{array}{c}60 \text { patients } \\
(\text { LPV } / \text { RTV }=20, \\
\text { Huashi Baidu } \\
\text { Formula }+ \\
\text { LPV } / \text { RTV }=20, \\
\text { Huashi Baidu } \\
\text { Formula }=20)\end{array}$ & Oral & $\begin{array}{l}\text { No significant difference was } \\
\text { observed for clinical } \\
\text { remission rate between the } 3 \\
\text { groups }(95 \%(19 / 20), 100 \% \\
(20 / 20) \text {, and } 100 \%(20 / 20)) \text {. }\end{array}$ & NA & Warrants larger RCT \\
\hline $\mathrm{LPV} / \mathrm{RTV}$ & Cao et al. [81] & $\begin{array}{c}\text { RCT } \\
\text { (ChiCTR2000029308) }\end{array}$ & China & $\begin{array}{c}199 \text { patients }(T=99 \\
\text { and } C=100)\end{array}$ & Oral & $\begin{array}{c}\text { No significant difference } \\
\text { between LPV / RTV and SOC } \\
\text { was observed in clinical } \\
\text { improvement or } \\
\text { mortality rate. }\end{array}$ & $\begin{array}{l}\text { GI ADRs were more } \\
\text { common in the } \\
\text { treatment group. }\end{array}$ & $\begin{array}{l}\text { No significant } \\
\text { difference was } \\
\text { observed between } \\
\text { the two groups. }\end{array}$ \\
\hline LPV/RTV, ARB & Li et al. [82] & $\begin{array}{c}\text { RCT } \\
\text { (NCT04252885) }\end{array}$ & China & $\begin{array}{l}44 \text { patients } \\
(\mathrm{LPV} / \mathrm{RTV}=21, \\
\mathrm{ARB}=16 \text {, and } \\
\mathrm{SOC}=7)\end{array}$ & Oral & $\begin{array}{l}\text { No significant difference } \\
\text { between groups in } \\
\text { antipyresis, cough alleviation, } \\
\text { or improvement of chest } \\
\text { computed tomography (CT) }\end{array}$ & $\begin{array}{l}12 \text { and } 5 \text { patients were } \\
\text { suffering from ADRs in } \\
\text { the LPV/RTV and ARB } \\
\text { groups, respectively. }\end{array}$ & $\begin{array}{l}\text { No significant } \\
\text { benefit was observed } \\
\text { among these three } \\
\text { groups. }\end{array}$ \\
\hline $\begin{array}{l}\mathrm{LPV} / \mathrm{RTV}+ \\
\mathrm{RBV}+\mathrm{IFN} \\
\text { beta-1b }\end{array}$ & $\begin{array}{l}\text { Hung } \\
\text { et al. [83] }\end{array}$ & RCT (NCT04276688) & China & $\begin{array}{l}127 \text { (combination } \\
\text { group } 86 \text { and control } \\
(\mathrm{LPV} / \mathrm{RTV})=41)\end{array}$ & $\begin{array}{l}\text { Oral, } \\
\text { SC }\end{array}$ & $\begin{array}{c}\text { The combination group } \\
\text { showed a significantly } \\
\text { shorter recovery time than } \\
\text { the control ( } 7 \text { days vs. } \\
12 \text { days, respectively; } \mathrm{HR}= \\
4.37,95 \% \mathrm{CI}=1.86-10.24 ; \\
p=0.0010) .\end{array}$ & $\begin{array}{c}\text { Diarrhea } \\
(52(41 \%)) \text {, fever } \\
(48(38 \%)) \text {, nausea }(43 \\
(34 \%)) \text {, and raised } \\
\text { alanine } \\
\text { transaminase } \\
\text { level }(18(14 \%))\end{array}$ & $\begin{array}{c}\text { Early triple } \\
\text { combination therapy } \\
\text { showed lower } \\
\text { recovery time with } \\
\text { safety than only } \\
\text { LPV/RTV. }\end{array}$ \\
\hline $\begin{array}{l}\mathrm{LPV} / \mathrm{RTV}+ \\
\text { IFN-a }\end{array}$ & $\begin{array}{l}\text { Huang } \\
\text { et al. [84] }\end{array}$ & $\begin{array}{c}\text { RCT } \\
\text { (ChiCTR2000029387) }\end{array}$ & China & $\begin{array}{c}101 \text { patients } \\
(\mathrm{LPV} / \mathrm{RTV}+ \\
\text { IFN- } \alpha=36, \mathrm{RBV}+ \\
\text { IFN- } \alpha=33, \text { and } \\
\mathrm{LPV} / \mathrm{RTV}+\mathrm{RBV}+ \\
\text { IFN- } \alpha+=32\end{array}$ & Oral & $\begin{array}{l}\text { No statistically significant } \\
\text { differences in viral clearance } \\
\text { among these } 3 \text { groups } \\
\text { (LPV /RTV + IFN-a vs. RBV + } \\
\text { IFN-a vs. RBV + LPV + IFN-a } \\
=12 \text { days vs. } 13 \text { days vs. } 15 \\
\text { days, respectively; } p=0.23 \text { ) }\end{array}$ & $\begin{array}{l}\text { Adverse gastrointestinal } \\
\text { events were higher for } \\
\text { the LPV / RTV + RBV + } \\
\text { IFN-a group than the } \\
\text { LPV / RTV + IFN-a and } \\
\text { RBV + IFN-a groups }\end{array}$ & $\begin{array}{c}\text { No significant } \\
\text { difference was } \\
\text { observed among the } \\
\text { three groups. }\end{array}$ \\
\hline
\end{tabular}


Table 1. Cont.

\begin{tabular}{|c|c|c|c|c|c|c|c|c|}
\hline Drug & Reference & Study Type & Country & $n$ & ROA & Results & ADR & Interpretation \\
\hline $\mathrm{LPV} / \mathrm{RTV}$ & $\begin{array}{c}\text { RECOVERY } \\
\text { Collaborative } \\
\text { Group [85] }\end{array}$ & $\begin{array}{c}\text { RCT } \\
\text { (NCT04381936) }\end{array}$ & UK & $\begin{array}{c}5040 \text { patients } \\
(\mathrm{LPV} / \mathrm{RTV}=1616 \\
\text { and standard of } \\
\text { care }=3424)\end{array}$ & Oral & $\begin{array}{c}\text { No significant difference at } \\
28 \text {-day fatality rate } \\
\text { (LPV/RTV vs. SOC = } 23 \% \text { vs. } \\
22 \% \text {, respectively; } p=0.60 \text { ) }\end{array}$ & NA & $\begin{array}{l}\text { The treatment is not } \\
\text { effective for } \\
\text { COVID-19. }\end{array}$ \\
\hline $\begin{array}{l}\mathrm{LPV} / \mathrm{RTV}+ \\
\text { recombinant IFN } \\
\text { beta- } 1 \mathrm{~b}\end{array}$ & $\begin{array}{c}\text { Arabi } \\
\text { et al. [86] }\end{array}$ & $\begin{array}{c}\text { RCT } \\
\text { (NCT02845843) }\end{array}$ & Saudi Arabia & $\begin{array}{l}95 \text { patients } \\
(\mathrm{LPV} / \mathrm{RTV}+ \\
\text { recombinant IFN } \\
\text { beta- } 1 \mathrm{~b}=43 \text { and } \\
\text { placebo }=52)\end{array}$ & $\begin{array}{l}\text { Oral, } \\
\text { SC }\end{array}$ & $\begin{array}{l}\text { Intervention therapy against } \\
\text { MERS-CoV led to lower } \\
\text { fatality rate ( } R R=0.19 ; 95 \% \\
C I=0.05 \text { to } 0.75) \text { than } \\
\text { placebo }\end{array}$ & $\begin{array}{c}\text { Serious ADRs: } \\
\text { Intervention } \\
(4(9 \%)) \text { and placebo } \\
(10(19 \%))\end{array}$ & $\begin{array}{l}\text { Further study with } \\
\text { larger sample size }\end{array}$ \\
\hline CQ+ LPV/RTV & $\begin{array}{l}\text { Sevilla- } \\
\text { Castillo } \\
\text { et al. [87] }\end{array}$ & Retrospective & Mexico & $\begin{array}{c}61(\mathrm{LPV} / \mathrm{RTV}=27 \\
\mathrm{CQ}=11 \\
\text { combination }=17 \\
\text { and } \mathrm{C}=6)\end{array}$ & Oral & $\begin{array}{l}\text { Both the drugs were } \\
\text { ineffective in COVID } \\
\text { treatment; combination } \\
\text { therapy enhanced mortality. }\end{array}$ & $\begin{array}{c}\text { Increase in lactate } \\
\text { dehydrogenase and } \\
\text { ferritin }\end{array}$ & $\begin{array}{l}\text { Warrants further } \\
\text { investigation }\end{array}$ \\
\hline $\begin{array}{c}\text { IFN beta } 1 b+ \\
\text { LPV / RTV + RBV } \\
\text { vs. FPV }\end{array}$ & $\begin{array}{l}\text { Malhani } \\
\text { et al. [88] }\end{array}$ & Cohort & Saudi Arabia & $\begin{array}{c}222(\text { IFN triple } \\
\text { therapy }=68 \text { and } \\
\text { FPV }=154)\end{array}$ & $\begin{array}{l}\text { Oral, } \\
\text { SC }\end{array}$ & $\begin{array}{l}\text { IFN-based triple therapy } \\
\text { decreased the mortality rate } \\
\text { in non-critical patients. }\end{array}$ & Nausea and diarrhea & Warrants RCT \\
\hline $\mathrm{LPV} / \mathrm{RTV}$ & $\begin{array}{l}\text { Lepage } \\
\text { et al. [89] }\end{array}$ & Retrospective & Canada & 12 & Oral & $\begin{array}{l}\text { Trough concentration is } \\
\text { higher when an HIV dose } \\
\text { regimen is used; dose } \\
\text { tampering might be needed } \\
\text { to avoid risk in } \\
\text { COVID-19 patients. }\end{array}$ & $\begin{array}{l}\text { GI symptoms, electrolyte } \\
\text { imbalance, liver enzyme } \\
\text { disturbance, and } \\
\text { TG elevation }\end{array}$ & Warrants RCT \\
\hline $\begin{array}{l}\mathrm{LPV} / \mathrm{RTV}+ \\
\mathrm{HCQ}\end{array}$ & $\begin{array}{l}\text { Schneider } \\
\text { et al. [90] }\end{array}$ & Retrospective & Germany & $\begin{array}{c}79(\text { Non-ICU- } \\
\text { LPV } / \text { RTV + HCQ }= \\
14 \text { and C = } 14 ; \text { ICU- } \\
\text { LPV } / \text { RTV = } 30 \text { and } \\
4 \mathrm{C}=21)\end{array}$ & $\begin{array}{l}\text { Not men- } \\
\text { tioned }\end{array}$ & $\begin{array}{l}\text { Triple therapy may cause } \\
\text { elevated acute kidney injury } \\
\text { in non-severe } \\
\text { COVID patients. }\end{array}$ & $\begin{array}{l}\text { Slight hematuria and } \\
\text { proteinuria }\end{array}$ & Warrants RCT \\
\hline $\begin{array}{c}\text { IFN-k + TFF2 }+ \\
\text { SOC }\end{array}$ & Fu et al. [91] & $\begin{array}{c}\text { RCT } \\
\text { (ChiCTR2000030262) }\end{array}$ & China & $\begin{array}{c}80 \text { patients } \\
(\text { experimental } \\
\text { group }=40 \text { and } \\
\text { control group }=40)\end{array}$ & inhalation & $\begin{array}{l}\text { The experimental group } \\
\text { exhibited significantly } \\
\text { shorter days in viral RNA } \\
\text { (-ve) conversion than the } \\
\text { control group ( } 3.8 \text { vs. } \\
7.40 \text { days, respectively). }\end{array}$ & $\begin{array}{l}\text { No discomfort or } \\
\text { complication } \\
\text { was reported. }\end{array}$ & $\begin{array}{l}\text { The experimental } \\
\text { combination with } \\
\text { SOC is safe and } \\
\text { superior to } \\
\text { SOC alone. }\end{array}$ \\
\hline
\end{tabular}


Table 1. Cont.

\begin{tabular}{|c|c|c|c|c|c|c|c|c|}
\hline Drug & Reference & Study Type & Country & $n$ & ROA & Results & ADR & Interpretation \\
\hline $\begin{array}{l}\text { rSIFN vs. } \\
\text { traditional } \\
\text { IFN-alpha }\end{array}$ & Li et al. [92] & $\mathrm{RCT}$ & China & $\begin{array}{l}94(\mathrm{rSIFN}=46 \text { and } \\
\text { IFN-alpha }=48)\end{array}$ & $\begin{array}{l}\text { Nasal } \\
\text { (Nebuliza- } \\
\text { tion) }\end{array}$ & $\begin{array}{c}\text { Shortened the clinical } \\
\text { improvement time (rSIFN vs. } \\
\text { INF-alpha }=11.5 \text { days vs. } 14 \\
\text { days, respectively), clinical } \\
\text { improvement rate }(\mathrm{rSIFN}= \\
93.5 \%, \text { IFN-alpha }=77.1 \%), \\
\text { radiological improvement } \\
\text { time (rSIFN = } 8 \text { days, } \\
\text { IFN-alpha }=10 \text { days), and } \\
\text { viral shedding time (rSIFN = } \\
7 \text { days, IFN-alpha }=10 \text { days) }\end{array}$ & Decreased appetite & $\begin{array}{c}\text { rSIFN was associated } \\
\text { with shorter time of } \\
\text { clinical } \\
\text { improvement. } \\
\text { However, a } \\
\text { broader-range trial is } \\
\text { needed. }\end{array}$ \\
\hline $\begin{array}{l}\text { IFN- alpha + } \\
\text { RBV }\end{array}$ & Li et al. [93] & Retrospective cohort & China & $\begin{array}{c}2037(\mathrm{RBV}=840, \\
\text { IFN-alpha }=214, \\
\text { RBV }+ \\
\text { IFN-alpha }=227, \text { and } \\
\text { C }=756)\end{array}$ & NA & $\begin{array}{l}\text { No significant difference in } \\
\text { mortality rate between the } \\
\text { two groups }\end{array}$ & $\begin{array}{l}\text { Decreased hemoglobin } \\
\text { and increased uric acid }\end{array}$ & $\begin{array}{l}\text { Should avoid this } \\
\text { combination in the } \\
\text { treatment of } \\
\text { COVID-19 }\end{array}$ \\
\hline $\begin{array}{l}\text { IFN-beta 1a vs. } \\
\text { IFN-beta } 1 \mathrm{~b}\end{array}$ & $\begin{array}{l}\text { Darazam } \\
\text { et al. [94] }\end{array}$ & $\mathrm{RCT}$ & Iran & $\begin{array}{c}60(\text { IFN-beta } 1 \mathrm{a}=20 \\
\text { IFN-beta } 1 \mathrm{~b}=20 \\
\text { and } \mathrm{C}=20)\end{array}$ & SC & $\begin{array}{l}\text { Improved time to clinical } \\
\text { improvement in IFN-beta } \\
\text { 1a group }\end{array}$ & Liver injury, ARDS & $\begin{array}{l}\text { Warrants larger } \\
\text { studies }\end{array}$ \\
\hline $\begin{array}{c}\mathrm{ARB}+ \\
\mathrm{LPV} / \mathrm{RTV}\end{array}$ & Wen et al. [96] & Cohort & China & 178 & NA & $\begin{array}{l}\text { Significant difference in } \\
\text { proportion of deterioration } \\
\text { changing from } \\
\text { mild / moderate to } \\
\text { severe/critical type at day } 7 \\
\text { was found }\end{array}$ & $\begin{array}{l}\text { ADRs were higher than } \\
\text { the SOC group. }\end{array}$ & $\begin{array}{l}\text { Warrants larger } \\
\text { studies }\end{array}$ \\
\hline $\begin{array}{c}\mathrm{ARB}+ \\
\mathrm{LPV} / \mathrm{RTV}\end{array}$ & Deng et al. [97] & Cohort & China & $\begin{array}{l}33 \text { patients }(\mathrm{ARB}+ \\
\mathrm{LPV} / \mathrm{RTV}=16 \text { and } \\
\mathrm{LPV} / \mathrm{RTV}=17)\end{array}$ & Oral & $\begin{array}{c}\text { Observed significant } \\
\text { difference in SARS-CoV-2 } \\
\text { negativity between ARB }+ \\
\text { LPV / RTV and LPV /RTV } \\
\text { groups at day } 7 \text { ( } 75 \% \text { vs. } 35 \% \text {; } \\
p<0.05 \text { ) and day } 14 \text { ( } 94 \% \text { vs. } \\
52.9 \% \text {, respectively) }\end{array}$ & $\begin{array}{l}\text { Digestive upset }(43.7 \%) \\
\text { such as mild diarrhea } \\
\text { and nausea }\end{array}$ & Warrants RCT \\
\hline
\end{tabular}


Table 1. Cont.

\begin{tabular}{|c|c|c|c|c|c|c|c|c|}
\hline Drug & Reference & Study Type & Country & $n$ & ROA & Results & ADR & Interpretation \\
\hline $\begin{array}{l}\text { ARB }+ \text { Shufeng } \\
\text { Jiedu capsules }\end{array}$ & Chen et al. [98] & Cohort & China & $\begin{array}{c}200 \\
(\text { experimental = } 100 \\
\text { and control }=100)\end{array}$ & Oral & $\begin{array}{l}\text { The experimental group } \\
\text { showed significantly more } \\
\text { efficacy in pneumonia } \\
\text { resolution thank the control } \\
\text { group }(p<0.05)\end{array}$ & $\begin{array}{l}\text { Nausea, allergic reaction, } \\
\text { abdominal pain, and } \\
\text { diarrhea were common } \\
\text { for both groups. }\end{array}$ & Warrants RCT \\
\hline $\begin{array}{l}\text { ARB + Shufeng } \\
\text { Jiedu capsules }\end{array}$ & Qu et al. [99] & Observational & China & $\begin{array}{l}70 \text { patients (ARB }+ \\
\text { Shufeng Jiedu } \\
\text { capsules }=40 \text { and } \\
\text { ARB group }=30 \text { ) }\end{array}$ & Oral & $\begin{array}{l}\text { The combination group } \\
\text { (ARB + Shufeng Jiedu } \\
\text { capsules) reduced negative } \\
\text { conversion time significantly } \\
\text { more than the ARB group } \\
\qquad(p<0.05) .\end{array}$ & NA & $\begin{array}{l}\text { The combination } \\
\text { therapy was better } \\
\text { than the ARB alone. }\end{array}$ \\
\hline $\begin{array}{l}\mathrm{ARB}+\mathrm{LQ} \\
\text { granules }\end{array}$ & Yu et al. [100] & Observational & China & $\begin{array}{c}295 \text { patients } \\
\text { (observation group }= \\
147 \text { and control } \\
\text { group }=14 \text { ) }\end{array}$ & Oral & $\begin{array}{c}\text { Effective rate was } \\
\text { significantly higher in } \\
\text { observation group than } \\
\text { control group ( } 80.95 \% \text { vs. } \\
64.86 \% \text {, respectively) }\end{array}$ & NA & $\begin{array}{l}\text { Warrants further } \\
\text { intensive study }\end{array}$ \\
\hline $\begin{array}{c}\mathrm{ARB}+ \\
\text { moxifloxacin }\end{array}$ & Xi et al. [101] & Cohort & China & 94 & NA & $\begin{array}{c}\text { Treatment with ARB + } \\
\text { moxifloxacin reduced viral } \\
\text { load and inflammation. }\end{array}$ & NA & $\begin{array}{l}\text { Warrants further } \\
\text { clinical verification }\end{array}$ \\
\hline $\mathrm{ARB}+\mathrm{IFN}-\mathrm{a} 2 \mathrm{~b}$ & Xu et al. [103] & Non-RCT & China & 141 & Oral & $\begin{array}{l}\text { No significant difference was } \\
\text { reported in viral load } \\
\text { clearance and hospital stay } \\
\text { duration between ARB + } \\
\text { IFN-a2b and IFNa-2b }\end{array}$ & NA & Warrants RCT \\
\hline $\begin{array}{l}\text { ARB vs. } \\
\text { KALETRA }\end{array}$ & $\begin{array}{l}\text { Nojomi } \\
\text { et al. [104] }\end{array}$ & $\begin{array}{c}\text { RCT (IRCT201807250 } \\
\text { 40596N2) }\end{array}$ & Iran & $\begin{array}{c}100 \text { patients } \\
(\mathrm{ARB}=50 \text { and } \\
\text { KALETRA }=50)\end{array}$ & Oral & $\begin{array}{l}\text { ARB significantly reduced } \\
\text { hospital stay duration } \\
\text { compared to the KALETRA } \\
\text { arm }(7.2 \text { vs. } 9.6 \text { days, } \\
\text { respectively; } p=0.02)\end{array}$ & Nausea and vomiting & $\begin{array}{l}\text { Warrants more } \\
\text { studies with larger } \\
\text { sample size }\end{array}$ \\
\hline
\end{tabular}


Table 1. Cont.

\begin{tabular}{|c|c|c|c|c|c|c|c|c|}
\hline Drug & Reference & Study Type & Country & $n$ & ROA & Results & ADR & Interpretation \\
\hline $\mathrm{ARB}+\mathrm{HCQ}$ & $\begin{array}{l}\text { Ghaderkhani } \\
\text { et al. [105] }\end{array}$ & $\mathrm{RCT}$ & Iran & $\begin{array}{c}56 \text { patients, } 3 \\
\text { patients left from } \\
\text { ARB group; ARB } \\
(n=28) \text { and control } \\
(n=25) \text { arms }\end{array}$ & NA & $\begin{array}{l}\text { After } 7 \text { days, the ARB + HCQ } \\
\text { group showed significantly } \\
\text { faster recovery in dry cough } \\
(p=0.001) \text {, weakness } \\
(p=0.004), \text { gastrointestinal } \\
\text { symptoms }(p=0.043), \text { and } \\
\text { shortness of breath }(p=0.001) \\
\text { than the control group. }\end{array}$ & $\begin{array}{c}\text { Dermatitis, GI symptoms } \\
\text { (nausea and diarrhea), } \\
\text { jaundice, and } \\
\text { neurological symptoms } \\
\text { were not observed in any } \\
\text { of the patients after } \\
14 \text { days. }\end{array}$ & $\begin{array}{l}\text { The combination } \\
\text { group showed a } \\
\text { better effect in the } \\
\text { recovery process. }\end{array}$ \\
\hline ARB vs. CQ & Li et al. [106] & Retrospective & China & $\begin{array}{c}62(\mathrm{ARB}=42 \text { and } \\
\mathrm{CQ}=20)\end{array}$ & NA & $\begin{array}{l}\text { Length of hospital stay was } \\
\text { significantly lower for the } \\
\text { ARB than the HCQ group. }\end{array}$ & $\begin{array}{l}\text { Vomiting, hepatic } \\
\text { function impairment, } \\
\text { and ALT elevation }\end{array}$ & Warrants RCT \\
\hline $\begin{array}{l}\text { Oseltamivir + } \\
\text { antibacterial } \\
\text { agents } \\
\text { (levofloxacin }+ \\
\text { garenoxacin) }\end{array}$ & $\begin{array}{l}\text { Chiba } \\
\text { et al. [107] }\end{array}$ & Cohort & Japan & 16 patients & Oral & $\begin{array}{l}\text { Early treatment with } \\
\text { oseltamivir shortened fever } \\
\text { duration time compared to } \\
\text { the late treatment }(31 \pm 21 \mathrm{~h} \\
\text { vs. } 94 \pm 38 \mathrm{~h} \text {, respectively; } \\
\quad p<0.001) .\end{array}$ & $\begin{array}{l}\text { Mild side effects } \\
\text { were present. }\end{array}$ & $\begin{array}{l}\text { Early use of } \\
\text { oseltamivir might } \\
\text { reduce fever } \\
\text { duration. }\end{array}$ \\
\hline $\begin{array}{l}\text { DRV / RTV + } \\
\text { HCQ }\end{array}$ & $\begin{array}{l}\text { Meriglier } \\
\text { et al. [108] }\end{array}$ & Cohort & France & $\begin{array}{c}46 \text { patients }(\mathrm{HCQ}+ \\
\mathrm{DRV} / \mathrm{RTV}=25 \text { and } \\
\mathrm{HCQ}+ \\
\mathrm{LPV} / \mathrm{RTV}=21)\end{array}$ & Oral & $\begin{array}{l}\text { Combination therapy led to } \\
\text { severe illness. }\end{array}$ & $\begin{array}{c}\text { ECG abnormalities } \\
(n=4), \text { repolarization } \\
\text { disorder }(n=3), \\
\text { conduction disorder } \\
(n=1), \text { diarrhea grade } \\
\mathrm{I} / \mathrm{II}(n=8), \text { and hepatic } \\
\text { enzyme increased }(n=1)\end{array}$ & $\begin{array}{l}\text { Concomitant use } \\
\text { was not safe. }\end{array}$ \\
\hline $\begin{array}{l}\text { INF beta-1a vs. } \\
\text { medications of } \\
\text { national } \\
\text { protocols }\end{array}$ & $\begin{array}{l}\text { Davoudi- } \\
\text { Monfared } \\
\text { et al. [109] }\end{array}$ & $\begin{array}{c}\text { RCT } \\
\text { (IRCT20100228003449N28) }\end{array}$ & Iran & $\begin{aligned} 81(\mathrm{~T} & =42 \text { and } \\
\mathrm{C} & =39)\end{aligned}$ & SC & $\begin{array}{l}\text { Significantly improved the } \\
\text { discharge rate and } \\
\text { decreased the } 28 \text {-day } \\
\text { mortality rate. }\end{array}$ & $\begin{array}{l}\text { For the IFN beta-1a } \\
\text { group: IFN-related } \\
\text { injection reactions } \\
(19.04), \text { neuropsychiatric } \\
\text { problems }(9.52 \%)\end{array}$ & $\begin{array}{l}\text { Needs more } \\
\text { extensive study }\end{array}$ \\
\hline $\begin{array}{l}\text { Novaferon }+ \\
\text { LPV/RTV }\end{array}$ & Qu et al. [110] & Case-control study & China & $\begin{array}{c}170(\text { male }=81 \text { and } \\
\text { female }=89)\end{array}$ & NA & $\begin{array}{l}\text { Novaferon + LPV / RTV } \\
\text { exerted a lower duration of } \\
\text { hospital stay and negative } \\
\text { nucleic acid conversion } \\
\text { compared to LPV/RTV or } \\
\text { combination with IFN } \\
\text { or ARB. }\end{array}$ & NA & $\begin{array}{l}\text { Novaferon }+ \\
\text { LPV / RTV may have } \\
\text { better efficacy than } \\
\text { these control groups. } \\
\text { Warrants RCT }\end{array}$ \\
\hline
\end{tabular}




\subsection{Favipiravir (FPV)}

Favipiravir (FPV, T-705, 6-fluoro-3-hydroxypyrazine-2-carboxamide), a pyrazine ana$\log$, is another broad-spectrum RNA-dependent RNA polymerase (RdRp) enzyme inhibitor that has been used in an extended pandemic platform to treat patients with COVID-19 in various countries around the world [111,112].

Cai et al. [43] performed an open-level, controlled trial on 80 COVID-19 patients to evaluate the safety and efficacy of FPV in comparison with lopinavir-ritonavir (LPV/RTV) as a control. In this study, 35 patients were subjected to FPV treatment $(1600 \mathrm{mg}$ on the first day and $600 \mathrm{mg}$ twice daily for the next 19 days), 45 patients to LPV/RTV treatment (400 mg/100 mg twice daily), and both groups also received 5 million units of interferon (IFN)- $\alpha$ aerosol inhalation twice daily. In chest imaging, computed tomography (CT) demonstrated a substantial improvement rate with FPV compared to the LPV/RTV group $(91.43 \%$ vs. $62.22 \%$, respectively; $p=0.004)$ with several adverse consequences. FPV displayed relatively better therapeutic upshots in viral load reduction along with slower disease progression in this open-level controlled study. A randomized, multicenter, openlevel phase 3 trial was undertaken on 150 patients $(\mathrm{FPV}=75$ and control $=75)$ with mild to moderate severity of COVID-19 in India by Glenmark Pharmaceuticals. After 4 days, FPV exhibited statistically noteworthy upshots in refining clinical symptoms compared to the control group ( $69.8 \%$ vs. $44.9 \%$, respectively) [46]. Recently, a clinical trial on 50 patients with COVID-19 in Bangladesh (Dhaka trial) was accomplished, where FPV demonstrated significant viral clearance following 10 days of treatment (FPV $=96 \%$ and placebo $=52 \%$ ) with a good safety profile [47]. Another retrospective cohort study conducted by Alamer et al. [49] showed that FPV reduced the median time of hospital discharge (FPV and SOC $=10$ days vs. 15 days, respectively) and was associated with less progression to mechanical ventilation. However, the drug did not significantly influence the mortality rate of severe cases [49]. Zhao et al. [50] conducted a multicenter, open-labeled randomized trial on 55 SARS-CoV-2 re-positive patients (FPV $=36$ and SOC $=19$ ). They revealed that the FPV shortened the duration of viral shedding and significantly decreased C-reactive protein (CRP) levels compared to the control group.

An additional study performed on 150 patients with mild to moderate COVID-19 (FPV and control $=1: 1$ ) reported that the drug significantly reduced the required median time to cessation of viral termination ( 5 days vs. 7 days, respectively) and clinical cure time ( 3 days vs. 5 days, respectively). However, the FPV group of patients showed more adverse drug reactions compared to the control group ( $36 \%$ vs. $8 \%$, respectively) [51]. Another study reported in Turkey that a 31-year-old female receiving FPV treatment developed some neurotic disorders that were suspected to be caused by the drug [112]. Despite possessing excellent therapeutic profiles, FPV showed embryotoxic and teratogenic effects. Therefore, the Japanese Drug Safety Bureau provided strong warnings regarding the use of FPV in pregnant women [113]. Additionally, Kaur et al. [114] assessed the adverse drug events (ADEs) of FPV by analyzing the WHO pharmacovigilance data set and concluded that FPV reduced inflammatory markers as well as offered clinical improvement for critically ill patients.

Moreover, an open-level, randomized control trial conducted by Khamis et al. [72] found no significant difference in therapeutic output of the drug plus inhaled interferon beta- $1 \mathrm{~b}$ in the treatment of hospitalized moderate-to-severe COVID-19 patients. Chen et al. [44] performed an open-level, multicenter, and randomized controlled trial on 240 patients $(\mathrm{FPV}=120$, Arbidol $(\mathrm{ARB})=120)$ with COVID-19 and reported that FPV showed shorter latency recovery from fever and cough. Additionally, Lou et al. [45] conducted a randomized trial (ChiCTR2000029544) on 30 patients (baloxavir marboxil $=10$, $\mathrm{FPV}=10$, and control $=10$ ) with COVID-19. After the 14 days of the experiment, the percentage of cessation of viral shedding was $70 \%, 77 \%$, and $100 \%$, respectively. Due to lack of time from the onset of symptoms to randomization, the baloxavir marboxil, and control groups did not confer clinical benefits against SARS-CoV-2 compared to the FPV group. Another retrospective study reported that the patients who received hydroxy- 
chloroquine (HCQ) alongside early treatment of FPV showed more PCR negativity and slower progression of the disease [48]. However, Dabbous et al. [69] reported no significant difference among HCQ, FPV, and their combination therapy groups regarding beneficial effects against COVID-19.

\subsection{Interferons (IFNs)}

IFNs (alpha, beta, and gamma) are naturally occurring groups of cytokines that serve as natural barriers against foreign viruses, bacteria, mitogens, and tumor cell infections. When they are bound to the target receptors, a variety of genes become activated, which accounts for antiviral and antiproliferation, and immunomodulation activities [115]. The US FDA approved IFN- $\beta$ to treat multiple sclerosis and hepatitis B and C infections [116]. A deficiency state of interferon might elicit the SARS-CoV-2 infection, and several clinical trials have been started since the inception phase of the pandemic to evaluate the safety and efficacy of IFNs against COVID-19 [117].

An open-label prospective clinical trial was conducted by Meng et al. [52] to investigate the safety and efficacy of rhIFN- $\alpha$ (recombinant human interferon-alpha) nasal drops with or without thymosin- $\alpha 1$ on medical professionals. The authors concluded that the rhIFN- $\alpha$ strictly protected medical staff with the augmented safeguards. A randomized multicenter clinical trial was conducted by Li et al. [92] to evaluate the efficacy of recombinant super compound-interferon (rSIFN-co) with traditional IFN in COVID-19 treatment, and the rSINF group showed better clinical improvement time (rSIFN $=11.5$ days, IFN-alpha $=14$ days), clinical improvement rate $(\mathrm{rSIFN}=93.5 \%$, IFN-alpha $=77.1 \%)$, radiological improvement time (rSIFN $=8$ days, IFN-alpha $=10$ days), and viral shedding time ( $\mathrm{rSIFN}=7$ days, IFN-alpha $=10$ days). Another randomized control trial was reported wherein IFN-alpha $2 b$ exerted greater efficacy in the duration of hospitalization and viral clearance [53]. In another retrospective study in China on 1401 patients with COVID-19 ( $\mathrm{T}=852$ and $\mathrm{C}=549)$, Yu et al. [54] showed improved viral clearance and decreased hospital stay by using IFN-alpha $2 \mathrm{~b}$. In addition, IFN-lambda resulted in accelerated viral decline and better clinical improvement in a randomized control trial [55]. Additionally, Yuan et al. [118] assessed that the combination therapeutic regimens of IFN- $\alpha$ with LPV/RTV or ribavirin or both options displayed favorable responses in contrast to COVID-19 infection. During the treatment of 77 hospitalized SARS-CoV-2-infected patients with IFN- $\alpha 2 b$ with or without arbidol (ARB), Zhou et al. [119] found a reduced duration of viral loading in the upper respiratory tract as well as less duration of inflammatory markers in the blood. In a multicenter retrospective cohort study, Wang et al. [120] exhibited that early treatment with IFN- $\alpha 2 \mathrm{~b}$ decreased hospital fatality, although late treatment with IFN was associated with higher fatality in the case of patients with COVID-19.

Another randomized controlled trial (IRCT20100228003449N28) on 81 patients with severe COVID-19 [109] assessed the efficacy and safety of IFN beta-1a compared with a control group ( $n=39$; taking medications only according to national guidelines). Although the primary result displayed no significant difference between the IFN and the control groups $(p=0.95)$ in time to clinical response, early administration of IFN beta-1a greatly improved the discharge rate (odds ratio (OR), 2.5; 95\% confidence interval (CI), 1.05 to 6.37) on day 14 and decreased the 28 -day mortality rate (treatment vs. control $=19 \%$ vs. $43.6 \%$, respectively; $p=0.015$ ). Fu et al. [91] conducted another randomized, open-level clinical trial (ChiCTR2000030262) on 80 patients (experimental group $=40$ and control group $=40$ ) to evaluate the safety and efficacy of the combination of IFN-k plus TFF2 with standard of care (SOC) and SOC alone. In comparison between the two groups, the experimental group shortened the viral RNA negative conversion time more than the control group ( 3.80 days, $95 \% \mathrm{CI}=2.07$ to 5.53 vs. 7.40 days, $95 \% \mathrm{CI}=4.57$ to 10.23 , respectively; $p=0.031$ ). $\mathrm{Li}$ et al. [93] compared the efficacy among IFN-alpha, ribavirin (RBV), and the combination $(\mathrm{IFN}$-alpha $=240, \mathrm{RBV}=814$, combination $=227$, and $\mathrm{C}=756)$ in a retrospective cohort study, and no significant clinical improvement was observed in the combination group. Therefore, the authors suggested that this combination needs to be avoided during the 
treatment of COVID-19. In another randomized controlled clinical trial $(n=60 ;$ IFN $\beta 1 \mathrm{a}=$ IFN $\beta 1 b=$ control = 20), Darazam et al. [94] found that IFN $\beta-1$ a had a significant difference in time to clinical improvement compared to the control group. In contrast, INF $\beta-1 b$ exerted no significant influence on time to clinical improvement.

\subsection{Lopinavir-Ritonavir (LPV/RTV)}

Lopinavir (LPV) is usually prescribed for the prevention of human immunodeficiency virus (HIV) as well as, previously, SARS-CoV and MERS-CoV infections, in combination with a lower dose of a ritonavir (RTV) booster. Mechanistically, LPV acts as a peptidomimetic inhibitor of the HIV-1 protease enzyme, whereas RTV plays a key role as a pharmacokinetics promoter of LPV by slowing down its hepatic metabolism of the CYP3A4 enzyme, and thus increasing the plasma concentration and plasma half-life of LPV [121,122]. Because of its potential effect on viral loading at the cellular stage, initial therapy of LPV/RTV in COVID-19 demonstrated a decrease in steroid usage and nosocomial infections depending on the SARS and MARS experiences [123].

In a retrospective cohort study, Lee et al. [74] compared the clinical improvement of LPV / RTV with HCQ in patients with mild to moderate COVID-19. In this study, the clinical failure rate was more common in the HCQ group than in the LPV/r group $(41 \%(11 / 27)$ vs. $2 \%(1 / 45)$, respectively; $p=0.001)$ and the disease progression rate was $44 \%(12 / 27)$ and $18 \%(8 / 45)$, respectively $(p=0.030)$. Finally, the authors [74] concluded that LPV / RTV was superior to HCQ in preventing the disease progression to severe COVID-19. Another cohort study carried out by Yu et al. [75] measured the effect of LPV/RTV on 128 enrolled COVID19 patients divided into influenza A/B coinfected $(n=64)$ and non-infected $(n=64)$ groups. In this retrospective study, LPV/RTV displayed a significant difference in the median duration of viral shedding time in patients with influenza compared to non-influenza (17.0 vs. 12.0 days, respectively; $p<0.001)$. Among the patients with coinfected with influenza, those who were treated with LPV/RTV showed pneumonia resolution within two weeks after the onset of symptoms ( $37 \%$ vs. $1 \%$, respectively; $p=0.001)$. Another open-level randomized controlled platform clinical trial [85] on 5040 hospitalized COVID19 patients $(\mathrm{SOC}=3424$ and $\mathrm{LPV} / \mathrm{RTV}=1616)$ presented no significant difference in the 28 -day fatality rate $(\mathrm{LPV} / \mathrm{RTV}$ vs. SOC $=23 \%$ vs. $22 \%$, respectively rate ratio $=1.03,95 \%$ $\mathrm{CI}=0.91-1.17 ; p=0.60$ ). In this study, LPV / RTV did not improve clinical outcomes by minimizing hospital stay, mechanical ventilation, or mortality rate. A retrospective study was conducted to observe whether the conventional dose of LPV/RTV used for HIV can be used in COVID-19 patients; it turns out dose tampering is a mandatory act to use the drug for the latter purpose [89]. Cao et al. [74] conducted a randomized, controlled, open-label clinical trial (ChiCTR2000029308) on 199 patients (LPV / RTV $=99$ and SOC $=100)$. Even though clinical improvement was observed (hazard ratio $(\mathrm{HR})=1.13,95 \% \mathrm{CI}=0.95,1.80$ ), no significant difference was found in mortality rate at day 28 (LPV / RTV vs. SOC $=19.2 \%$ vs. $25 \%$, respectively, absolute difference: $-5.8,95 \% \mathrm{CI}=-17.3,-5.7)$.

Moreover, Kim et al. [73] conducted a retrospective study on 65 patients (LPV/RTV $=31$, HCQ = 34) with mild to moderate COVID-19, and reported that LPV/RTV exhibited shorter viral clearance time (adjusted hazard ratio (aHR), 2.28; 95\% confidence interval (CI), 1.24 to 4.21 ) than HCQ treatment. However, no significant difference in clinical improvement was observed between the groups (median: 18 days vs. 21 days, respectively). In contrast, lymphopenia and hyperbilirubinemia were more frequent in the LPV/RTV group than the control group. Lecronier et al. [76] conducted a retrospective study on 80 patients with COVID-19 divided into three groups $(\mathrm{SOC}=22, \mathrm{LPV} / \mathrm{r}+\mathrm{SOC}=20$, and $\mathrm{HCQ}+\mathrm{SCQ}=38$ patients). At day 28 , treatment escalation was observed (SOC $=41 \%$, $\mathrm{LPV} / \mathrm{r}+\mathrm{SOC}=50 \%, \mathrm{HCQ}+\mathrm{SOC}=39 \% ; p=0.567)$ and no substantial difference was observed between these three groups in 28 ventilator-free days and the 14-day fatality rate.

In a study conducted by Lan et al. [77], the patients receiving lopinavir-ritonavir or an arbidol combination (LPV / RTV + ARB) displayed a 4.8\% higher remedy rate and 1.5-day hospital stay compared to those who received LPV/RTV therapy only. Another retro- 
spective study [78] conducted on a total of 129 non-severe COVID-19 patients (SOC $=59$, $\mathrm{LPV} / \mathrm{RTV}=51$, and chloroquine, $\mathrm{CQ}=19$ ) found no significant difference in the improvement of prognosis of disease or shortening the clinical course. Similarly, in another cohort study on 156 hospitalized patients (median age, 72 years (IQR: 55.25-81)) with severe COVID-19 to compare the safety and efficacy of LPV/RTV $(n=47)$ with HCQ $(n=20)$, Karolyi et al. [79] observed no significant differences in mortality rate $(8.5 \%$ vs. $15 \%$, respectively; $p=0.418)$, ICU admission rate $(12.8 \%$ vs. $20 \%$, respectively; $p=0.47)$, or hospital stay (11 days vs. 9 days, respectively; $p=0.34$ ).

In a non-randomized controlled trial, LPV/RTV showed a clinically effective and safety index for the enrolled COVID-19 patients. Here, 60 patients were involved in three groups $(\mathrm{LPV} / \mathrm{RTV}=20$, Huashi Baidu Formula $+\mathrm{LPV} / \mathrm{RTV}=20$, Huashi Baidu Formula $=20)$ and their clinical remission rates were $95 \%(19 / 20), 100 \%(20 / 20)$, and $100 \%$ $(20 / 20)$, respectively. No significant differences were found among these three groups. However, these results urgently need to be confirmed further by well-designed prospective double-blinded randomized control clinical trials [80]. In addition, Li et al. [82] conducted a randomized control trial (NCT04252885) on 86 patients $(\mathrm{LPV} / \mathrm{RTV}=34, \mathrm{ARB}=35$, and control $=17$ ) with mild to moderate COVID-19. The difference in the median time of the negative conversion rate of SARS-CoV-2 nucleic acid, antipyresis, cough alleviation, and improvement of chest computed tomography (CT) was not significant $(p>0.05)$.

Hung et al. [83] conducted a randomized, open-level, prospective, multicenter, phase II, controlled trial (NCT04276688) on 127 (combination group = 82 and control group = 41) hospitalized patients with COVID-19. The trial, randomized at a 2:1 ratio for 14 days, involved triple combination group therapy named LPV/RTV $(400 \mathrm{mg} / 100 \mathrm{mg}$ twice daily), IFN beta-1b (3 doses of 8 million IU on alternate days), and ribavirin (400 $\mathrm{mg}$ twice daily), and the control group was LPV/RTV (400 mg/100 mg twice daily). The required median time for negative COVID-19 results from the day of the onset of treatment for the combination group and control group was 7 days (IQR $=5-11)$ and 12 days $(\mathrm{IQR}=8-15)$, respectively (hazard ration (HR) 4.37 (95\% CI 1.86-10.24), $p=0.0010)$. Triple combination therapy depicted significantly lower recovery median time together with greater safety and efficacy than the control group with no mortality during the study. Huang et al. [84] performed an open-level, randomized, single-center, prospective clinical trial (ChiCTR2000029387) on patients with mild to moderate severity of COVID-19 divided into three groups (ribavirin (RBV) + I-a: LPV $/ \mathrm{RTV}+\mathrm{IFN}-\mathrm{a}$ : ribavirin $+\mathrm{LPV} / \mathrm{r}+\mathrm{IFN}-\mathrm{a}=$ 1:1:1). In this analysis, the median time of positive-to-negative conversion of SARS-CoV-2 nucleic acid in LPV/RTV + IFN-a, RBV + IFN-a, and RBV + LPV + IFN-a groups was $61 \%$, $51.5 \%$, and $46.9 \%$, respectively $(p=0.23$ ). Though this result showed no significant difference among these three groups, the combination therapy of RBV and LPV/RTV offered a significant increase in gastrointestinal adverse consequences. So, combination therapy of $\mathrm{RBV}$ and LPV/r should not be relied on in COVID-19 patients. Arabi et al. [86] conducted a randomized, placebo-controlled, double-blinded adaptive clinical trial (NCT02845843) on 95 patients infected with MERS-CoV (intervention (LPV/RTV + recombinant IFN beta$1 b)=43$ and placebo $=52$ ) in nine sites in Saudi Arabia. In this analysis, the primary outcome at day 90 that accounted for the adaptive design yielded a risk difference of $19 \%$. Intervention treatment contributed to a reduced probability of fatality (relative risk 0.19 ; $95 \%$ CI, 0.05 to 0.75 ) than the placebo with serious adverse responses, such as increased levels of liver enzymes.

A retrospective analysis found that both CQ and LPV/RTV showed ineffectiveness in the case of severe COVID-19, whereas a combination of them enhanced mortality [87]. Another cohort study of non-critical COVID-19 patients receiving FPV $(n=154)$ and triple therapy (INF-beta $1 b+$ RBV + LPV / RTV) showed that the triple therapy decreased mortality [88]. Additionally, Qu et al. [110] assessed the therapeutic efficacy of LPV/RTV alone or in combination with IFN, novaferon, and arbidol against 170 COVID-19 patients (male $=81$ and female $=89$ ), which resulted in the promising favorable consequence of LPV /RTV combined with novaferon than LPV/RTV alone or any combinations (LPV/RTV + IFN or 
LPV/RTV + IFN + arbidol, LPV/RTV + IFN + novaferon). Choi et al. [124] reported no significant difference in viral shedding between HCQ and LPV/RTV groups. A comparative randomized clinical trial between sofosbuvir/daclatasvir (SFV/DCV) and LPV/RTV was carried out where only the discharge rate was better in the LPV/RTV group; better survival rate and fewer complications were shown in the SFV/DCV group [125]. Similarly, Lepage et al. [89] and Schneider et al. [90] conducted retrospective studies and found several preliminary results that warrant well-designed and more extensive randomized controlled clinical trials.

\subsection{Arbidol (ARB)}

Umifenovir, popularly known as arbidol (ARB), is a drug of choice in the prophylactic treatment of the influenza virus. It has recently been recommended against COVID-19 due to its therapeutic benefits against the SARS-CoV-2 infection [126]. From the outset, Wang et al. [127] showed that ARB had profound efficiency in inhibiting SARS-CoV-2 infection by interfering with virus entry ( $75 \%$ inhibition) to host cells and post-entry ( $55 \%$ inhibition) by blocking intracellular vesicle trafficking. Several clinical studies have been started to investigate its safety and efficacy against COVID-19.

Zhang et al. [128] performed a retrospective cohort study on 66 members in 27 families and 124 health care workers who were close to COVID-19 patients. The study reported that ARB could minimize the infection risk of SARS-CoV-2. Likewise, a cohort study explained that $\mathrm{ARB}$ treatment progressed the hospital discharge rate and decreased the mortality rate by $7.5 \%$. [56]. In addition, $\mathrm{Xu}$ et al. [129] described that ARB accelerated the viral clearance and is clinically effective for COVID-19 infection. Moreover, prophylactic therapy of ARB could reduce the incidence of COVID-19 infection but not the hospitalization rate in health care providers [61]. Additionally, a retrospective cohort study (ChiCTR2000030931) carried out by Huang et al. [102] noted that ARB reduced the viral shedding interval and declined the hospitalization duration rate. In another retrospective cohort study, ARB showed decreased fatality rate in severe COVID-19 patients [62]. Additionally, Deng et al. [97] reported that the combination therapy of ARB with LPV/RTV demonstrated significant efficiency compared to LPV / RTV monotherapy in terms of viral clearance at the 7th day ( $75 \%$ vs. $35 \%$, respectively; $p<0.05$ ). Furthermore, another retrospective cohort study [98] reported that the combination therapy (Shufeng Jiedu capsules + ARB) had notable clinical safety, efficacy, and a higher recovery time compared to the control therapy.

Moreover, a retrospective non-randomized multicenter cohort study [103] concluded that ARB/IFN-2b could be prescribed to ameliorate COVID-19 pneumonia in mild patients, though the therapy was ineffective at accelerating virus clearance. In addition, an openlabel controlled clinical trial [104] observed that ARB significantly improved clinical and laboratory conditions compared to LPV/RTV. Additionally, Zhu et al. [58] recommended that ARB monotherapy has more effectiveness in viral clearance compared to LPV/RTV. Another study [59] demonstrated that adjuvant therapy combined with ARB might relieve fever and accelerate the recovery time. Moreover, another study [100] found faster curative efficacy by using a combined treatment of ARB along with Lianhua Qingwen granules ( $80.95 \%$ vs. $64.86 \%$, respectively; $p<0.05)$. In addition, studies have shown that ARB and moxifloxacin therapy in severe COVID-19 patients is correlated with lowering the viral load and fatal inflammation [101]. Another retrospective cohort study was conducted to compare the efficacy of ARB, LPV/RTV, and oseltamivir, wherein the ARB group dominated due to its lower fatality rate and higher viral clearance [60]. Furthermore, Li et al. [106] concluded that ARB was superior to HCQ in terms of duration of hospital stay and SARS-CoV-2 negative conversion. Moreover, a randomized control trial proved the higher benefit in using ARB over HCQ, as it was responsible for the improvement of clinical symptoms and recovery rate [105].

A retrospective cohort study conducted by Fang et al. [95] manifested that combined medication (traditional Chinese medicine called Lianhuaqingwen + ARB) did not accelerate the recovery of severe COVID-19 patients. Similarly, a retrospective observational 
study (ChiCTR2000030391) [99] concluded that the combined medication (ARB + Shufeng Jiedu Capsules) did not show any noticeable effect compared to a single administration. Another study conducted by Yadegarinia et al. [130] assessed that ARB could not cure severely infected patients, but it was effective in mild to moderate conditions. Similarly, Chen et al. [131] reported that the antiviral medications did not shorten the clearance of viral RNA in acute patients. Moreover, Wen et al. [96] found that ARB combined with $\mathrm{LPV} / \mathrm{RTV}$ could not improve clinical condition and did not reduce the time to convert the nucleic acid of SARS-CoV-2 to negative. Likewise, Lian et al. [57] discovered that ARB might not accelerate viral clearance, and treatment led to a longer hospitalization rate compared to standard care. Furthermore, a randomized controlled multicenter trial (ChiCTR2000030254) [44] concluded that ARB did not show any notable recovery rate at day $7(\mathrm{FPV}=71 / 116$ and $\mathrm{ARB}=62 / 120 ; p=0.1396)$. So far, a variety of randomized phase IV clinical studies, for instance, NCT04260594, NCT04255017, NCT04254874, NCT04350684, and so on, are now under investigation to evaluate the safety and efficacy of ARB combination therapy or a standard/control therapy.

\subsection{Oseltamivir}

Oseltamivir, commonly known as Tamiflu, is a first-line antiviral treatment formerly used to treat and prevent flu A and flu B. It inhibits the distribution of neuraminidase on the surface of the influenza virus in the human body [132,133]. An initial study in Wuhan reported that several patients were treated with oseltamivir as an empirical therapy before SARS-CoV-2 was detected, but no positive result was found [134]. Although oseltamivir monotherapy has not shown any significant effect against COVID-19 [135], numerous clinical trials are still taking it into consideration to evaluate the efficacy of oseltamivir against COVID-19 infection [136].

An observational study showed that early exposure to oseltamivir significantly reduced ventilation duration, hospital/ICU stay, and overall fatality rate compared to late exposure among critically ill patients with influenza pneumonia [64]. Tan et al. [63] conducted a retrospective analysis on 333 patients with COVID-19 and reported that oseltamivir significantly reduced hospital stay $(p=0.0096)$ over other groups (LPV/RTV, $\mathrm{ARB}$, corticosteroids). This trial also showed that a combined treatment of oseltamivir with hydroxychloroquine might be prudent in patients with COVID-19. Chiba et al. [107] performed another study that included 16 healthcare providers suspected of having COVID-19 and their cohabitation families without hypoxia. The authors concluded that early administration of oseltamivir plus antibacterial therapy might reduce the duration of fever in outpatients suspected to have COVID-19 without hypoxia.

On the other hand, a real-world observational study [137] performed on 395,343 patients led to the observation that oseltamivir is associated with increased mortality in the general population ( $\mathrm{HR}=1.72,95 \% \mathrm{CI}: 1.61-1.84)$, ambulatory patients $(\mathrm{HR}=4.79,95 \% \mathrm{CI}$ : 4.01-5.75), non-critical patients ( $\mathrm{HR}=2.05,95 \% \mathrm{CI}: 1.88-2.23)$, and pregnant patients $(\mathrm{HR}=8.35,95 \% \mathrm{CI}: 1.77-39.30)$, as well as hospitalized (HR $=1.13,95 \% \mathrm{CI}: 1.01-1.26)$ and critical patients (HR $=1.22,95 \%$ CI:1.05-1.43). Similarly, Chen et al. [131] conducted another cohort study on 284 patients and described that oseltamivir did not shorten viral RNA clearance, particularly in non-serious patients. Moreover, several clinical trials (for instance, NCT04303299, NCT04516915, NCT04338698, and so on) are ongoing to evaluate the effectiveness of oseltamivir in treating SARS-CoV-2 infection.

\subsection{Darunavir (DRV)}

Darunavir (DRV) is a second-generation protease inhibitor extensively used to treat HIV infection with cobicistat as a pharmacokinetic booster [138,139]. Several studies reported that DRV could inhibit COVID-19 enzymatic proteins by inhibiting the chymotrypsinlike protease of SARS-CoV-2 [139,140].

Deng et al. [65] evaluated the safety and efficacy of darunavir/cobicistat (DRV/c) in COVID-19-infected patients in Zhongnan Hospital of Wuhan University. They worked 
on a total of 66 patients $(\mathrm{DRV} / \mathrm{c}=32$ and control group $=34)$ and found that the nucleic acid conversion duration was significantly shorter in the DRV/c group from the onset of symptoms to admission within three days $(7.9 \pm 6.7$ days $)$ than above three days (15.9 \pm 7.1 days) $(p=0.01)$. Meriglier et al. [108] conducted another study on 46 patients $(\mathrm{HCQ}+\mathrm{DRV} / \mathrm{RTV}=25$ and HCQ + LPV $/ \mathrm{RTV}=21)$ and found that the combination therapy of HCQ and DRV / RTV showed ECG abnormalities, mostly in older (age $=70$ ) patients with hypertension, chronic cardiovascular disease, and kidney failure.

In another report, Kim et al. [66] observed 110 critically ill hospitalized SARS-CoV-2infected patients (DRV-c group $=14$ and control $=96)$ in Korea. After 1:2 propensity-score matched analysis, DRV-c group also showed a lower fatality rate than the controls (OR $0.07,95 \%$ CI $0.01-0.52, p=0.009$ ). Chen et al. [67] conducted a randomized open-label trial $($ NCT04252274) on 30 patients (DRV/c = 15 and control $=15)$ with SARS-CoV-2-infected patients. The difference of negative PCR conversion rate in between two groups at day 7 was $13.3 \%$ (DRV/c vs. control $=9 / 15(60.0 \%)$ vs. $7 / 15(46.7 \%)$, respectively; $p=0.72)$. At day 3, viral clearance was $20 \%(3 / 15)$ in both groups and at day 5 , the difference of negative PCR conversion rate between the two groups was $6.7 \%$ (DRV/c vs. control $=26.7 \%(4 / 15)$ vs. $20 \%(3 / 15)$, respectively).

\section{Discussion and Concluding Remarks}

The new emergence of such a deadly virus needs potential agents to control it. It can be noted that mass vaccination is not easily affordable in many developing and underdeveloped countries immediately or before 2024 [141]. Additionally, over-populated countries might face some critical situations to ensure vaccines for all people due to vaccine insufficiency, and it might take longer to eradicate the outbreak. Moreover, the delta variant of concern has shown to be several times more resistant against vaccine-induced immunity [142]. This crucial situation calls for effective treatment tools/antiviral agents to fight against the deadly infectious disease. This review mainly focuses on the efficiency and adverse effects of repurposing antiviral drugs potentially used to treat COVID-19.

Various drugs are being used as repurposing drugs, as there is no drug or effective treatment strategy against COVID-19. Therefore, the most promising seven antiviral drugs were taken under consideration to evaluate their efficacy and also their adverse effects in various levels of COVID-19 patients.

The very first drug and most widely used one was RDV. Though several studies mentioned above showed reduced mortality, shorter hospital stay, and better improvement of symptoms, several studies have been reported it to be insignificant in the case of COVID19 patients' recovery. Notably, the Macaque experiment stated that RDV was only efficient in viral clearance from the lower respiratory tract, not the upper one [143]. Another randomized controlled study conducted by Wang et al. [37] suggested no efficacy of RDV on the respiratory tract, neither upper nor lower. We can conclude by evaluating the above data that RDV might be effective for severe patients in mitigating the fatality rate and improvement of clinical conditions. The WHO has recommended RDV against in non-severe patients, which might match with the current findings.

FPV has shown great efficacy in this crucial situation, yet it is not patient-friendly due to its high dose. The studies discussed above revealed that FPV is a potential cure for COVID-19, both in severe and mild cases. Chen et al. [44] reported a positive effect of FPV compared to ARB, as the former showed better improvement of symptoms like cough, pyrexia, and difficulty breathing. The drug is beneficial in alleviating symptoms, reducing viral load and hospital stay, but still, it is considered risky due to severe side effects like teratogenicity and embryotoxicity [144]. This drug was very effective against H1N1 virus, which is also an RNA virus and has shown potential antiviral effects, perhaps most efficient in viral clearance. There has been no toxicity detected for its huge loading dose, but some adverse effects have been reported; cardiovascular complications, nausea, vomiting, diarrhea, and kidney injuries are the common ones. Several studies also compared the efficacy among FPV, hydroxychloroquine, chloroquine, and IFN, which had minimal 
significant difference. LPV / RTV combination was also a drug of choice for COVID-19 in the early stages of the pandemic. However, this combination proved to be insignificant in most of the cases studied, with some exceptions. The combination rather became the reason behind serious complications like diarrhea, gastrointestinal disturbance, electrolyte imbalance, and severe acute kidney injury in patients with both severe and mild COVID-19.

INF therapy is one of the promising treatments for COVID-19 now. Several subcategories of INF are known, such as IFN-alpha 1a, IFN-alpha 1b, IFN-beta 1a, IFN-beta $1 \mathrm{~b}$, IFN-gamma, and IFN-lambda. All have shown a variety of efficacy without severe side effects. They have worked to the betterment of patients' condition and reduced the fatality rate among severe patients. Several studies were also carried out to evaluate and compare the potentials of these variants. Recombinant IFN has proven to be more potent than traditional ones. An efficient therapy known as triple therapy is a combination of IFN $/ \mathrm{LPV} / \mathrm{r} / \mathrm{RBV}$ that has shown amazing results. Type I interferons (IFN-I, IFN- $\alpha$, and IFN- $ß$ ) are immunomodulatory drugs that can induce antiviral effects and may induce pro-inflammatory activity, which has turned out to be beneficial for early treatment of COVID-19 [145]. Combination with other antiviral agents may mitigate the adverse effects as well as improve the clinical condition of the patients.

ARB has shown noteworthy effects in alleviating symptoms of COVID-19 and shortening the time for converting the PCR result from positive to negative. The potential antiviral agent works well both alone and in combination with various other agents like HCQ, RBV, and IFN. Antibiotic therapy alongside ARB is just the icing on the cake, showing prompt activity in quick recovery and reduction of fatality rate. It has proved to be more efficacious than other drugs like LPV/RTV and HCQ. All these have made it a potential agent in this pandemic situation. We can add oseltamivir to this list, but the drug showed great positive effects in severe patients when used early. Darunavir is emerging as a potent antiviral drug by lowering the mortality rate and hospital stay, but more studies are required to extensively verify its safety profile.

HCQ, LPV/RTV, and many other drugs, which were thought to be a treatment option earlier, are already banned by the WHO. FPV, ARB, and oseltamivir are in the pool of acceptable drugs for COVID-19, but there are still many randomized trials going on to assess their safety profiles. RDV is recommended in severe conditions considering its adverse effects that require limited use of the drug. Boosting the immune system is believed to be beneficial, so IFN type I might exert immunomodulation through its antiviral effects by stimulating interferon-stimulated gene (ISG).

As patients with COVID-19 might face multiple pathological dysfunctions, they must be prescribed multi-drug therapies [146]. Moreover, several pieces of evidence have demonstrated that combination therapy is superior to monotherapy, as the multiple drugs might heal by acting on multiple receptors [147]. Particularly, combination therapy in COVID-19 has become an excellent choice to wrestle against the disease. Several diagnoses revealed that secondary infections have become of paramount importance as an after-effect of COVID-19, and have some saddening consequences. A combination of potential antivirals with antibiotics is used to fight the secondary infections produced due to lessened immunity, along with the viral infection [148]. Interferon uses an immunomodulator that has served well for COVID-19, and its combination with antiviral agents gives the best results. Moreover, the combination lowers the time taken to reduce viral shedding significantly [149]. The mechanism could be the dual action of antivirals and immunomodulatory agents; one weakens the virus, and the latter strengthens the immunity so the body can recover faster, through confirmation or justification of this mechanism is yet to be provided. However, to define the exact mechanism and efficacy profile of the stated combinations in the current review, more precise investigation with larger data and evidence might be required.

Author Contributions: Conceptualization, M.J.H.; methodology, M.J.H., T.J., S.R.B., U.R., S.M., M.O.R., M.R.I., M.L.N., M.A.I. and T.B.E.; software, M.J.H., T.J., S.R.B., U.R., S.M., M.O.R., M.R.I., M.L.N., M.A.I. and T.B.E.; validation, M.J.H., T.J., S.R.B., U.R., S.M., M.O.R., M.R.I., M.L.N., M.A.I. 
and T.B.E.; formal analysis, M.J.H., T.J., S.R.B., U.R., S.M., M.O.R., M.R.I., M.L.N., M.A.I. and T.B.E.; investigation, M.J.H. and T.B.E.; resources, M.J.H., T.J., S.R.B., U.R., S.M., M.O.R., M.R.I., M.L.N., M.A.I. and T.B.E.; data curation, M.J.H., T.J., S.R.B., U.R., S.M., M.O.R., M.R.I., M.L.N., M.A.I. and T.B.E.; writing—original draft preparation, M.J.H., T.J., S.R.B., U.R. and S.M.; writing-review and editing, M.J.H., S.M., M.O.R., M.R.I., M.L.N., M.A.I. and T.B.E.; visualization, M.J.H., T.J., S.R.B., U.R., S.M., M.O.R., M.R.I., M.L.N., M.A.I. and T.B.E.; supervision, M.J.H. and T.B.E.; project administration, M.J.H. and T.B.E.; funding acquisition, T.B.E. All authors have read and agreed to the published version of the manuscript.

Funding: This work was conducted with the individual funding of all authors.

Institutional Review Board Statement: Not applicable.

Informed Consent Statement: Not applicable.

Data Availability Statement: Available data are presented in the manuscript and reference list.

Conflicts of Interest: The authors declare that they have no conflicts of interest.

\begin{tabular}{|c|c|}
\hline RDV & remdesivir \\
\hline FPV & favipiravir \\
\hline LPV-RTV & lopinavir-ritonavir \\
\hline INF & interferon \\
\hline $\operatorname{rhIFN}-\alpha$ & recombinant human interferon alpha \\
\hline rSIFN & recombinant super compound interferon \\
\hline TFF2 & trefoil factor 2 \\
\hline ARB & arbidol \\
\hline DRV & darunavir \\
\hline $\mathrm{DRV} / \mathrm{c}$ & darunavir/cobicisitat \\
\hline HCQ & hydroxychloroquine \\
\hline CQ & chloroquine \\
\hline $\mathrm{RCT}$ & randomized controlled trial \\
\hline LHQW & Lianhuaqingwen \\
\hline LQ & Lianhua Qingwen \\
\hline $\mathrm{CP}$ & convalescent plasma \\
\hline $\mathrm{BCN}$ & baricitinib \\
\hline RBV & ribavirin \\
\hline $\mathrm{SFV} / \mathrm{DCV}$ & sofosbuvir/daclatasvir \\
\hline $\mathrm{N}$ & number \\
\hline $\mathrm{T}$ & treatment (group) \\
\hline $\mathrm{C}$ & control (group) \\
\hline SOC & standard of care \\
\hline CT & computed tomography \\
\hline IV & intravenous \\
\hline NA & not available \\
\hline SC & subcutaneous \\
\hline GI & gastrointestinal \\
\hline $95 \%$ CI & $95 \%$ confidence interval \\
\hline HR & hazard ratio \\
\hline OR & odds ratio \\
\hline RR & relative risk \\
\hline ROA & route of administration \\
\hline ADR & adverse drug reaction \\
\hline ADRS & acute respiratory distress syndrome \\
\hline ALT & alanine aminotransferase \\
\hline $\operatorname{RdRp}$ & RNA-dependent RNA polymerase \\
\hline IQR & interquartile range \\
\hline PCR & polymerase chain reaction \\
\hline ECG & electrocardiogram \\
\hline
\end{tabular}




\section{References}

1. Ashraf, B.N. Economic impact of government interventions during the COVID-19 pandemic: International evidence from financial markets. J. Behav. Exp. Financ. 2020, 27, 100371. [CrossRef]

2. Hossain, M.J.; Ahmmed, F.; Rahman, S.; Sanam, S.; Emran, T.B.; Mitra, S. Impact of online education on fear of academic delay and psychological distress among university students following one year of COVID-19 outbreak in Bangladesh. Heliyon 2021, 7, e07388. [CrossRef]

3. Gollakner, R.; Capua, I. Is COVID-19 the first pandemic that evolves into a panzootic? Vet. Ital. 2020, 56, 11-12. [CrossRef]

4. Yoo, H.S.; Yoo, D. COVID-19 and veterinarians for one health, zoonotic- and reverse-zoonotic transmissions. J. Vet. Sci. 2020, 21, e51. [CrossRef] [PubMed]

5. Gupta, N.; Singhai, M.; Garg, S.; Shah, D.; Sood, V.; Singh, S.K. The missing pieces in the jigsaw and need for cohesive research amidst coronavirus infectious disease 2019 global response. Med. J. Armed Forces India 2020, 76, 132-135. [CrossRef] [PubMed]

6. Decaro, N.; Lorusso, A. Novel human coronavirus (SARS-CoV-2): A lesson from animal coronaviruses. Vet. Microbiol. 2020, 244, 108693. [CrossRef] [PubMed]

7. World Health Organization. Weekly Operational Update on COVID-19-20 July 2021; World Health Organization: Geneva, Switzerland, 2021; pp. 1-13.

8. Hossain, M.J.; Kuddus, M.R.; Rahman, S.M.A. Knowledge, attitudes, and behavioral responses toward COVID-19 during early phase in bangladesh: A questionnaire-based study. Asia Pac. J. Public Health 2021, 33, 141-144. [CrossRef] [PubMed]

9. Our World in Data. Statistics and Research. Coronavirus Disease 2019 (COVID-19) Vaccinations. Available online: https: / / ourworldindata.org/ covid-vaccinations (accessed on 30 August 2021).

10. Forni, G.; Mantovani, A.; COVID-19 Commission of Accademia Nazionale dei Lincei, Rome. COVID-19 vaccines: Where we stand and challenges ahead. Cell Death Differ. 2021, 28, 626-639. [CrossRef]

11. Centers for Disease Control and Prevention. Interim Clinical Guidance for Management of Patients with Confirmed Coronavirus Disease (COVID-19). Available online: https:/ /stacks.cdc.gov/view/cdc/88624 (accessed on 30 August 2021).

12. Hossain, M.J. Is Bangladesh moving toward herd immunity? Current COVID-19 perspective. Bangladesh J. Infect. Dis. 2020, 7 (Suppl. S2), S63-S66. [CrossRef]

13. Pushpakom, S.; Iorio, F.; Eyers, P.A.; Escott, K.J.; Hopper, S.; Wells, A.; Doig, A.; Guilliams, T.; Latimer, J.; McNamee, C.; et al. Drug repurposing: Progress, challenges and recommendations. Nat. Rev. Drug Discov. 2019, 18, 41-58. [CrossRef]

14. Hossain, M.J.; Kuddus, M.R.; Rashid, M.A.; Sultan, M.Z. Understanding and dealing the SARS-CoV-2 infection: An updated concise review. Bangladesh Pharm. J. 2021, 24, 61-75. [CrossRef]

15. Godwin, M.; Ruhland, L.; Casson, I.; MacDonald, S.; Delva, D.; Birtwhistle, R.; Lam, M.; Seguin, R. Pragmatic controlled clinical trials in primary care: The struggle between external and internal validity. BMC Med. Res. Methodol. 2003, 3, 28. [CrossRef] [PubMed]

16. Viveiros Rosa, S.G.; Santos, W.C. Clinical trials on drug repositioning for COVID-19 treatment. Rev. Panam. Salud Publica Pan Am. J. Public Health 2020, 44, e40. [CrossRef]

17. Wrapp, D.; Wang, N.; Corbett, K.S.; Goldsmith, J.A.; Hsieh, C.L.; Abiona, O.; Graham, B.S.; McLellan, J.S. Cryo-EM structure of the 2019-nCoV spike in the prefusion conformation. Science 2020, 367, 1260-1263. [CrossRef] [PubMed]

18. Lu, R.; Zhao, X.; Li, J.; Niu, P.; Yang, B.; Wu, H.; Wang, W.; Song, H.; Huang, B.; Zhu, N.; et al. Genomic characterisation and epidemiology of 2019 novel coronavirus: Implications for virus origins and receptor binding. Lancet 2020, 395, 565-574. [CrossRef]

19. Oprea, T.I.; Bauman, J.E.; Bologa, C.G.; Buranda, T.; Chigaev, A.; Edwards, B.S.; Jarvik, J.W.; Gresham, H.D.; Haynes, M.K.; Hjelle, B.; et al. Drug repurposing from an academic perspective. Drug Discov. Today Ther. Strateg. 2011, 8, 61-69. [CrossRef]

20. Senanayake, S.L. Drug repurposing strategies for COVID-19. Future Drug Discov. 2020, 2, FDD40. [CrossRef]

21. Young, B.E.; Ong, S.W.X.; Kalimuddin, S.; Low, J.G.; Tan, S.Y.; Loh, J.; Ng, O.-T.; Marimuthu, K.; Ang, L.W.; Mak, T.M.; et al. Epidemiologic Features and Clinical Course of Patients Infected With SARS-CoV-2 in Singapore. JAMA 2020, 323, 1488-1494. [CrossRef]

22. Yang, X.; Yu, Y.; Xu, J.; Shu, H.; Xia, J.; Liu, H.; Wu, Y.; Zhang, L.; Yu, Z.; Fang, M.; et al. Clinical course and outcomes of critically ill patients with SARS-CoV-2 pneumonia in Wuhan, China: A single-centered, retrospective, observational study. Lancet Respir. Med. 2020, 8, 475-481. [CrossRef]

23. Parvathaneni, V.; Gupta, V. Utilizing drug repurposing against COVID-19_Efficacy, limitations, and challenges. Life Sci. 2020, 259, 118275. [CrossRef]

24. Marra, F.; Smolders, E.J.; El-Sherif, O.; Boyle, A.; Davidson, K.; Sommerville, A.J.; Marzolini, C.; Siccardi, M.; Burger, D.; Gibbons, S.; et al. Recommendations for Dosing of Repurposed COVID-19 Medications in Patients with Renal and Hepatic Impairment. Drugs RED 2021, 21, 9-27. [CrossRef]

25. Hu, B.; Guo, H.; Zhou, P.; Shi, Z.L. Characteristics of SARS-CoV-2 and COVID-19. Nat. Rev. Microbiol. 2021, 19, 141-154. [CrossRef]

26. Zhou, Y.; Wang, F.; Tang, J.; Nussinov, R.; Cheng, F. Artificial intelligence in COVID-19 drug repurposing. Lancet Digit. Health 2020, 2, e667-e676. [CrossRef]

27. Sheahan, T.P.; Sims, A.C.; Leist, S.R.; Schäfer, A.; Won, J.; Brown, A.J.; Montgomery, S.A.; Hogg, A.; Babusis, D.; Clarke, M.O.; et al. Comparative therapeutic efficacy of remdesivir and combination lopinavir, ritonavir, and interferon beta against MERS-CoV. Nat. Commun. 2020, 11, 1-14. [CrossRef] 
28. Beigel, J.H.; Tomashek, K.M.; Dodd, L.E.; Mehta, A.K.; Zingman, B.S.; Kalil, A.C.; Hohmann, E.; Chu, H.Y.; Luetkemeyer, A.; Kline, S.; et al. Remdesivir for the Treatment of Covid-19-Final Report. N. Engl. J. Med. 2020, 383, 1813-1826. [CrossRef]

29. Grein, J.; Ohmagari, N.; Shin, D.; Diaz, G.; Asperges, E.; Castagna, A.; Feldt, T.; Green, G.; Green, M.L.; Lescure, F.-X.; et al. Compassionate Use of Remdesivir for Patients with Severe Covid-19. N. Engl. J. Med. 2020, 382, 2327-2336. [CrossRef]

30. Antinori, S.; Cossu, M.V.; Ridolfo, A.L.; Rech, R.; Bonazzetti, C.; Pagani, G.; Gubertini, G.; Coen, M.; Magni, C.; Castelli, A.; et al. Compassionate remdesivir treatment of severe Covid-19 pneumonia in intensive care unit (ICU) and Non-ICU patients: Clinical outcome and differences in post-treatment hospitalisation status. Pharmacol. Res. 2020, 158, 104899. [CrossRef]

31. Pasquini, Z.; Montalti, R.; Temperoni, C.; Canovari, B.; Mancini, M.; Tempesta, M.; Pimpini, D.; Zallocco, N.; Barchiesi, F. Effectiveness of remdesivir in patients with COVID-19 under mechanical ventilation in an Italian ICU. J. Antimicrob. Chemother. 2020, 75, 3359-3365. [CrossRef] [PubMed]

32. Aiswarya, D.; Arumugam, V.; Dineshkumar, T.; Gopalakrishnan, N.; Lamech, T.M.; Nithya, G.; Sastry, B.V.R.H.; Vathsalyan, P.; Dhanapriya, J.; Sakthirajan, R. Use of Remdesivir in Patients With COVID-19 on Hemodialysis: A Study of Safety and Tolerance. Kidney Int. Rep. 2021, 6, 586-593. [CrossRef]

33. Olender, S.A.; Perez, K.K.; Go, A.S.; Balani, B.; Price-Haywood, E.G.; Shah, N.S.; Wang, S.; Walunas, T.L.; Swaminathan, S.; Slim, J.; et al. Remdesivir for Severe Coronavirus Disease 2019 (COVID-19) Versus a Cohort Receiving Standard of Care. Clin. Infect. Dis. 2020, ciaa1041. [CrossRef] [PubMed]

34. Goldman, J.D.; Lye, D.C.B.; Hui, D.S.; Marks, K.M.; Bruno, R.; Montejano, R.; Spinner, C.D.; Galli, M.; Ahn, M.-Y.; Nahass, R.G.; et al. Remdesivir for 5 or 10 Days in Patients with Severe Covid-19. N. Engl. J. Med. 2020, 383, 1827-1837. [CrossRef]

35. Garibaldi, B.T.; Wang, K.; Robinson, M.L.; Zeger, S.L.; Roche, K.B.; Wang, M.-C.; Alexander, G.C.; Gupta, A.; Bollinger, R.; Xu, Y. Effectiveness of remdesivir with and without dexamethasone in hospitalized patients with COVID-19. medRxiv 2020. [CrossRef]

36. Tsuzuki, S.; Hayakawa, K.; Matsunaga, N.; Terada, M.; Suzuki, S.; Ohtsu, H.; Asai, Y.; Kitajima, K.; Saito, S.; Uemura, Y.; et al. Efficacy of remdesivir in Japanese patients hospitalised with COVID-19: A large observational study using the COVID-19 Registry Japan. medRxiv 2021. [CrossRef]

37. Wang, Y.; Zhang, D.; Du, G.; Du, R.; Zhao, J.; Jin, Y.; Fu, S.; Gao, L.; Cheng, Z.; Lu, Q.; et al. Remdesivir in adults with severe COVID-19: A randomised, double-blind, placebo-controlled, multicentre trial. Lancet 2020, 395, 1569-1578. [CrossRef]

38. Spinner, C.D.; Gottlieb, R.L.; Criner, G.J.; López, J.R.A.; Cattelan, A.M.; Viladomiu, A.S.; Ogbuagu, O.; Malhotra, P.; Mullane, K.M.; Castagna, A.; et al. Effect of Remdesivir vs. Standard Care on Clinical Status at 11 Days in Patients With Moderate COVID-19: A Randomized Clinical Trial. JAMA 2020, 324, 1048-1057. [CrossRef]

39. Kalil, A.C.; Patterson, T.F.; Mehta, A.K.; Tomashek, K.M.; Wolfe, C.R.; Ghazaryan, V.; Marconi, V.C.; Ruiz-Palacios, G.M.; Hsieh, L.; Kline, S.; et al. Baricitinib plus Remdesivir for Hospitalized Adults with Covid-19. N. Engl. J. Med. 2020, 384, 795-807. [CrossRef]

40. Falcão, F.; Viegas, E.; Carmo, I.; Soares, J.; Falcao, M.; Solano, M.; Cavaco, P.; Mendes, D.; Rijo, J.; Povoa, P.; et al. A prospective, observational study to evaluate adverse drug reactions in patients with COVID-19 treated with remdesivir or hydroxychloroquine: A preliminary report. Eur. J. Hosp. Pharm. 2021, 28, 248-253. [CrossRef]

41. Goldberg, E.; Zvi, H.B.; Sheena, L.; Sofer, S.; Krause, I.; Sklan, E.H.; Shlomai, A. A real-life setting evaluation of the effect of remdesivir on viral load in COVID-19 patients admitted to a large tertiary centre in Israel. Clin. Microbiol. Infect. 2021, 27, 917.e1-917.e4. [CrossRef]

42. Padilla, R.; Arquiette, J.; Mai, Y.; Singh, G.; Galang, K.; Liang, E. Clinical Outcomes of COVID-19 Patients Treated with Convalescent Plasma or Remdesivir Alone and in Combination at a Community Hospital in California's Central Valley. J. Pharm. Pharm. Sci. 2021, 24, 210-219. [CrossRef] [PubMed]

43. Cai, Q.; Yang, M.; Liu, D.; Chen, J.; Shu, D.; Xia, J.; Liao, X.; Gu, Y.; Cai, Q.; Yang, Y.; et al. Experimental Treatment with FPVipiravir for COVID-19: An Open-Label Control Study. Engineering 2020, 6, 1192-1198. [CrossRef] [PubMed]

44. Chen, C.; Zhang, Y.; Huang, J.; Yin, P.; Cheng, Z.; Wu, J.; Chen, S.; Zhang, Y.; Chen, B.; Lu, M.; et al. Favipiravir versus Arbidol for COVID-19: A Randomized Clinical Trial. medRxiv 2020. [CrossRef]

45. Lou, Y.; Liu, L.; Yao, H.; Hu, X.; Su, J.; Xu, K.; Luo, R.; Yang, X.; He, L.; Lu, X.; et al. Clinical Outcomes and Plasma Concentrations of Baloxavir Marboxil and FPVipiravir in COVID-19 Patients: An Exploratory Randomized, Controlled Trial. Eur. J. Pharm. Sci. 2021, 157, 105631. [CrossRef]

46. The Indian Express. COVID-19: Glenmark's FPVipiravir Shows Encouraging Results in Phase 3 Clinical Trial. Available online: https: / / www.newindianexpress.com/nation/2020/jul/23/covid-19-glenmarks-FPVipiravir-shows-encouraging-results-inphase-3-clinical-trial-2173500.html (accessed on 14 July 2021).

47. The Daily Star. Covid-19 Patients: Favipiravir Effective in Dhaka Trial. Available online: https://www.thedailystar.net/ backpage/news/covid-19-patients-FPVipiravir-effective-dhaka-trial-1927321 (accessed on 14 July 2021).

48. Ucan, A.; Cerci, P.; Efe, S.; Akgun, H.; Ozmen, A.; Yagmuroglu, A.; Bilgin, M.; Avci, D. Benefits of treatment with Favipiravir in hospitalized patients for COVID-19: A retrospective observational case-control study. Virol. J. 2021, 18, 102. [CrossRef] [PubMed]

49. Alamer, A.; Alrashed, A.A.; Alfaifi, M.; Alosaimi, B.; AlHassar, F.; Almutairi, M.; Howaidi, J.; Almutairi, W.; Mohzari, Y.; Sulaiman, T.; et al. Effectiveness and safety of Favipiravir compared to supportive care in moderately to critically ill COVID-19 patients: A retrospective study with propensity score matching sensitivity analysis. Curr. Med. Res. Opin. 2021, 37, 1085-1097. [CrossRef] 
50. Zhao, H.; Zhang, C.; Zhu, Q.; Chen, X.; Chen, G.; Sun, W.; Xiao, Z.; Du, W.; Yao, J.; Li, G.; et al. Favipiravir in the treatment of patients with SARS-CoV-2 RNA recurrent positive after discharge: A multicenter, open-label, randomized trial. Int. Immunopharmacol. 2021, 97, 107702. [CrossRef]

51. Udwadia, Z.F.; Singh, P.; Barkate, H.; Patil, S.; Rangwala, S.; Pendse, A.; Kadam, J.; Wu, W.; Caracta, C.F.; Tandon, M. Efficacy and safety of Favipiravir, an oral RNA-dependent RNA polymerase inhibitor, in mild-to-moderate COVID-19: A randomized, comparative, open-label, multicenter, phase 3 clinical trial. Int. J. Infect. Dis. 2021, 103, 62-71. [CrossRef] [PubMed]

52. Meng, Z.; Wang, T.; Chen, L.; Chen, X.; Li, L.; Qin, X.; Li, H.; Luo, J. The Effect of Recombinant Human Interferon Alpha Nasal Drops to Prevent COVID-19 Pneumonia for Medical Staff in an Epidemic Area. Curr. Top. Med. Chem. 2021, 21, $920-927$. [CrossRef]

53. Pandit, A.; Bhalani, N.; Bhushan, B.L.S.; Koradia, P.; Gargiya, S.; Bhomia, V.; Kansagra, K. Efficacy and safety of pegylated interferon alfa-2b in moderate COVID-19: A phase II, randomized, controlled, open-label study. Int. J. Infect. Dis. 2021, 105, 516-521. [CrossRef]

54. Yu, J.; Lu, X.; Tong, L.; Shi, X.; Ma, J.; Lv, F.; Wu, J.; Pan, Q.; Yang, J.; Cao, H.; et al. Interferon- $\alpha-2 b$ aerosol inhalation is associated with improved clinical outcomes in patients with coronavirus disease-2019. Br. J. Clin. Pharmacol. 2021, 2021, 1-10. [CrossRef]

55. Feld, J.J.; Kandel, C.; Biondi, M.J.; Kozak, R.A.; Zahoor, M.A.; Lemieux, C.; Borgia, S.M.; Boggild, A.K.; Powis, J.; McCready, J.; et al. Peginterferon lambda for the treatment of outpatients with COVID-19: A phase 2, placebo-controlled randomised trial. Lancet Respir. Med. 2021, 9, 498-510. [CrossRef]

56. Wang, Z.; Yang, B.; Li, Q.; Wen, L.; Zhang, R. Clinical Features of 69 Cases With Coronavirus Disease 2019 in Wuhan, China. Clin. Infect. Dis. 2020, 71, 769-777. [CrossRef]

57. Lian, N.; Xie, H.; Lin, S.; Huang, J.; Zhao, J.; Lin, Q. Umifenovir treatment is not associated with improved outcomes in patients with coronavirus disease 2019: A retrospective study. Clin. Microbiol. Infect. 2020, 26, 917-921. [CrossRef]

58. Zhu, Z.; Lu, Z.; Xu, T.; Chen, C.; Yang, G.; Zha, T.; Lu, J.; Xue, Y. Arbidol monotherapy is superior to lopinavir/ritonavir in treating COVID-19. J. Infect. 2020, 81, e21-e23. [CrossRef]

59. Chen, W.; Yao, M.; Fang, Z.; Lv, X.; Deng, M.; Wu, Z. A study on clinical effect of Arbidol combined with adjuvant therapy on COVID-19. J. Med. Virol. 2020, 92, 2702-2708. [CrossRef]

60. Liu, Q.; Fang, X.; Tian, L.; Vankadari, N.; Chen, X.; Wang, K.; Li, D.; Dai, X.; Xu, F.; Shen, L.; et al. Arbidol treatment with reduced mortality of adult patients with COVID-19 in Wuhan, China: A retrospective cohort study. medRxiv 2021. [CrossRef]

61. Yang, C.; Ke, C.; Yue, D.; Li, W.; Hu, Z.; Liu, W.; Hu, S.; Wang, S.; Liu, J. Effectiveness of Arbidol for COVID-19 Prevention in Health Professionals. Front. Public Health 2020, 8, 249. [CrossRef] [PubMed]

62. Zeng, H.; He, X.; Liu, W.; Kan, J.; He, L.; Zhao, J.; Chen, C.; Zhang, J.; Chen, S. Antiviral Abidol is Associated with the Reduction of In-Hospital Mortality in COVID-19 Patients. Cardiol. Discov. 2021, 1, 37-43.

63. Tan, J.; Yuan, Y.; Xu, C.; Song, C.; Liu, D.; Ma, D.; Gao, Q. A retrospective comparison of drugs against COVID-19. Virus Res. 2021, 294, 198262. [CrossRef]

64. Moreno, G.; Rodríguez, A.; Sole-Violán, J.; Martín-Loeches, I.; Díaz, E.; Bodí, M.; Reyes, L.F.; Gómez, J.; Guardiola, J.; Trefler, S.; et al. Early oseltamivir treatment improves survival in critically ill patients with influenza pneumonia. ERJ Open Res. 2021, 7, 00888-2020. [CrossRef]

65. Deng, L.; Xiong, Y.; Chen, T.; Zhang, Y.; Luo, M.; Gao, S.; Mo, P.; Hospital, Z.; Song, S.; Hospital Zhiyong, Z.; et al. Role of Darunavir/cobicisitat in the Treatment of COVID-19: Initial Virological and Clinical Findings. Res. Sq. 2021, $2021,1-23$. [CrossRef]

66. Kim, E.J.; Choi, S.H.; Park, J.S.; Kwon, Y.S.; Lee, J.; Kim, Y.; Lee, S.Y.; Choi, E.Y. Use of Darunavir-Cobicistat as a Treatment Option for Critically Ill Patients with SARS-CoV-2 Infection. Yonsei Med. J. 2020, 61, 826-830. [CrossRef]

67. Chen, J.; Xia, L.; Liu, L.; Xu, Q.; Ling, Y.; Huang, D.; Huang, W.; Song, S.; Xu, S.; Shen, Y.; et al. Antiviral Activity and Safety of Darunavir/Cobicistat for the Treatment of COVID-19. Open Forum Infect. Dis. 2020, 7, ofaa241. [CrossRef]

68. Guner, R.; Hasanoglu, I.; Kayaaslan, B.; Aypak, A.; Akinci, E.; Bodur, H.; Eser, F.; Kaya Kalem, A.; Kucuksahin, O.; Ates, I.; et al. Comparing ICU admission rates of mild/moderate COVID-19 patients treated with hydroxychloroquine, FPVipiravir, and hydroxychloroquine plus FPVipiravir. J. Infect. Public Health 2021, 14, 365-370. [CrossRef]

69. Dabbous, H.M.; Abd-Elsalam, S.; El-Sayed, M.H.; Sherief, A.F.; Ebeid, F.F.S.; El Ghafar, M.S.A.; Soliman, S.; Elbahnasawy, M.; Badawi, R.; Tageldin, M.A. Efficacy of Favipiravir in COVID-19 treatment: A multi-center randomized study. Arch. Virol. 2021, 166, 949-954. [CrossRef] [PubMed]

70. Kocayiğit, H.; Süner, K.Ö.; Tomak, Y.; Demir, G.; Yaylacı, S.; Dheir, H.; Güçlü, E.; Erdem, A.F. Observational study of the effects of FPVipiravir vs. Lopinavir/Ritonavir on clinical outcomes in critically Ill patients with COVID-19. J. Clin. Pharm. Ther. 2021, 46, 454-459. [CrossRef] [PubMed]

71. Dabbous, H.M.; El-Sayed, M.H.; El Assal, G.; Elghazaly, H.; Ebeid, F.F.S.; Sherief, A.F.; Elgaafary, M.; Fawzy, E.; Hassany, S.M.; Riad, A.R.; et al. Safety and efficacy of Favipiravir versus hydroxychloroquine in management of COVID-19: A randomised controlled trial. Sci. Rep. 2021, 11, 7282. [CrossRef]

72. Khamis, F.; Al Naabi, H.; Al Lawati, A.; Ambusaidi, Z.; Al Sharji, M.; Al Barwani, U.; Pandak, N.; Al Balushi, Z.; Al Bahrani, M.; Al Salmi, I.; et al. Randomized controlled open label trial on the use of FPVipiravir combined with inhaled interferon beta-1b in hospitalized patients with moderate to severe COVID-19 pneumonia. Int. J. Infect. Dis. 2021, 102, 538-543. [CrossRef] 
73. Kim, J.-W.; Kim, E.J.; Kwon, H.H.; Jung, C.Y.; Kim, K.C.; Choe, J.-Y.; Hong, H.-L. Lopinavir-ritonavir versus hydroxychloroquine for viral clearance and clinical improvement in patients with mild to moderate coronavirus disease 2019. Korean J. Intern. Med. 2020, 36, S253-S263. [CrossRef]

74. Lee, J.E.; Lee, S.O.; Heo, J.; Kim, D.W.; Park, M.R.; Son, H.; Kim, D.; Kim, K.-H.; Lee, S.; Lee, S.H. Comparative outcomes of lopinavir/ritonavir and hydroxychloroquine for the treatment of coronavirus disease 2019 with mild to moderate severity. Res. Sq. 2020, 2020, 1-20. [CrossRef]

75. Yu, C.; Zhang, Z.; Guo, Y.; Shi, J.; Pei, G.; Yao, Y.; Liao, W.; Zeng, R. Lopinavir/ritonavir is associated with pneumonia resolution in COVID-19 patients with influenza coinfection: A retrospective matched-pair cohort study. J. Med. Virol. 2021, 93, 472-480. [CrossRef]

76. Lecronier, M.; Beurton, A.; Burrel, S.; Haudebourg, L.; Deleris, R.; Le Marec, J.; Virolle, S.; Nemlaghi, S.; Bureau, C.; Mora, P.; et al Comparison of hydroxychloroquine, lopinavir/ritonavir, and standard of care in critically ill patients with SARS-CoV-2 pneumonia: An opportunistic retrospective analysis. Crit. Care 2020, 24, 1-9. [CrossRef]

77. Lan, X.; Shao, C.; Zeng, X.; Wu, Z.; Xu, Y. Lopinavir-ritonavir alone or combined with arbidol in the treatment of 73 hospitalized patients with COVID-19: A pilot retrospective study. Int. J. Clin. Pharmacol. Ther. 2021, 59, 378-385. [CrossRef]

78. Gao, G.; Wang, A.; Wang, S.; Qian, F.; Chen, M.; Yu, F.; Zhang, J.; Wang, X.; Ma, X.; Zhao, T.; et al. Brief Report: Retrospective Evaluation on the Efficacy of Lopinavir/Ritonavir and Chloroquine to Treat Nonsevere COVID-19 Patients. J. Acquir. Immune Defic. Syndr. 2020, 85, 239-243. [CrossRef]

79. Karolyi, M.; Pawelka, E.; Mader, T.; Omid, S.; Kelani, H.; Ely, S.; Jilma, B.; Baumgartner, S.; Laferl, H.; Ott, C.; et al. Hydroxychloroquine versus lopinavir/ritonavir in severe COVID-19 patients: Results from a real-life patient cohort. Wien. Klin. Wochenschr. 2020, 133, 284-291. [CrossRef]

80. Shi, N.; Guo, L.; Liu, B.; Bian, Y.; Chen, R.; Chen, S.; Chen, Y.; Chen, Y.; Cong, X.; Dong, G.; et al. Efficacy and safety of Chinese herbal medicine versus Lopinavir-Ritonavir in adult patients with coronavirus disease 2019: A non-randomized controlled trial. Phytomedicine 2021, 81, 153367. [CrossRef] [PubMed]

81. Cao, B.; Wang, Y.; Wen, D.; Liu, W.; Wang, J.; Fan, G.; Ruan, L.; Song, B.; Cai, Y.; Wei, M.; et al. A Trial of Lopinavir-Ritonavir in Adults Hospitalized with Severe Covid-19. N. Engl. J. Med. 2020, 382, 1787-1799. [CrossRef]

82. Li, Y.; Xie, Z.; Lin, W.; Cai, W.; Wen, C.; Guan, Y.; Mo, X.; Wang, J.; Wang, Y.; Peng, P.; et al. Efficacy and Safety of Lopinavir/Ritonavir or Arbidol in Adult Patients with Mild/Moderate COVID-19: An Exploratory Randomized Controlled Trial. Med 2020, 1, 105-113.e4. [CrossRef]

83. Hung, I.F.-N.; Lung, K.-C.; Tso, E.Y.-K.; Liu, R.; Chung, T.W.-H.; Chu, M.-Y.; Ng, Y.-Y.; Lo, J.; Chan, J.; Tam, A.R.; et al. Triple combination of interferon beta-1b, lopinavir-ritonavir, and ribavirin in the treatment of patients admitted to hospital with COVID-19: An open-label, randomised, phase 2 trial. Lancet 2020, 395, 1695-1704. [CrossRef]

84. Huang, Y.-Q.; Tang, S.-Q.; Xu, X.-L.; Zeng, Y.-M.; He, X.-Q.; Li, Y.; Harypursat, V.; Lu, Y.-Q.; Wan, Y.; Zhang, L.; et al. No Statistically Apparent Difference in Antiviral Effectiveness Observed Among Ribavirin Plus Interferon-Alpha, Lopinavir/Ritonavir Plus Interferon-Alpha, and Ribavirin Plus Lopinavir/Ritonavir Plus Interferon-Alpha in Patients With Mild to Moderate Coronavirus Disease 2019: Results of a Randomized, Open-Labeled Prospective Study. Front. Pharmacol. 2020, 11, 1071. [CrossRef]

85. Horby, P.W.; Mafham, M.; Bell, J.L.; Linsell, L.; Staplin, N.; Emberson, J.; Palfreeman, A.; Raw, J.; Elmahi, E.; Prudon, B.; et al. Lopinavir-ritonavir in patients admitted to hospital with COVID-19 (RECOVERY): A randomised, controlled, open-label, platform trial. Lancet 2020, 396, 1345-1352. [CrossRef]

86. Arabi, Y.M.; Asiri, A.Y.; Assiri, A.M.; Balkhy, H.H.; Al Bshabshe, A.; Al Jeraisy, M.; Mandourah, Y.; Azzam, M.H.A.; Bin Eshaq, A.M.; Al Johani, S.; et al. Interferon Beta-1b and Lopinavir-Ritonavir for Middle East Respiratory Syndrome. N. Engl. J. Med. 2020, 383, 1645-1656. [CrossRef]

87. Sevilla-Castillo, F.; Roque-Reyes, O.J.; Romero-Lechuga, F.; Gómez-Núñez, M.F.; Castillo-López, M.; Medina-Santos, D.; Román, P.O.; Flores-Hernández, J.R.; Méndez-Coca, J.D.; Montaño-Olmos, D.; et al. Both Chloroquine and Lopinavir/Ritonavir Are Ineffective for COVID-19 Treatment and Combined Worsen the Pathology: A Single-Center Experience with Severely Ill Patients. Biomed. Res. Int. 2021, 2021, 1-12. [CrossRef]

88. Malhani, A.A.; Enani, M.A.; Sharif-Askari, F.S.; Alghareeb, M.R.; Bin-Brikan, R.T.; AlShahrani, S.A.; Halwani, R.; Tleyjeh, I.M. Combination of (interferon beta-1b, lopinavir/ritonavir and ribavirin) versus FPVipiravir in hospitalized patients with non-critical COVID-19: A cohort study. PLoS ONE 2021, 16, e0252984. [CrossRef]

89. Lepage, M.-A.; Rozza, N.; Kremer, R.; Grunbaum, A. Safety and effectiveness concerns of lopinavir/ritonavir in COVID-19 affected patients: A retrospective series. Clin. Toxicol. 2021, 59, 644-647. [CrossRef] [PubMed]

90. Schneider, J.; Jaenigen, B.; Wagner, D.; Rieg, S.; Hornuss, D.; Biever, P.M.; Kern, W.V.; Walz, G. Therapy with lopinavir/ritonavir and hydroxychloroquine is associated with acute kidney injury in COVID-19 patients. PLoS ONE 2021, 16, e0249760. [CrossRef]

91. Fu, W.; Liu, Y.; Liu, L.; Hu, H.; Cheng, X.; Liu, P.; Song, Z.; Zha, L.; Bai, S.; Xu, T.; et al. An open-label, randomized trial of the combination of IFN-k plus TFF2 with standard care in the treatment of patients with moderate COVID-19. EClinicalMedicine 2020, 27, 100547. [CrossRef]

92. Li, C.; Luo, F.; Liu, C.; Xiong, N.; Xu, Z.; Zhang, W.; Yang, M.; Wang, Y.; Liu, D.; Yu, C.; et al. Effect of a genetically engineered interferon-alpha versus traditional interferon-alpha in the treatment of moderate-to-severe COVID-19: A randomised clinical trial. Ann. Med. 2021, 53, 391-401. [CrossRef] 
93. Li, H.; Xiong, N.; Li, C.; Gong, Y.; Liu, L.; Yang, H.; Tan, X.; Jiang, N.; Zong, Q.; Wang, J.; et al. Efficacy of ribavirin and interferon- $\alpha$ therapy for hospitalized patients with COVID-19: A multicenter, retrospective cohort study. Int. J. Infect. Dis. 2021, 104, 641-648. [CrossRef]

94. Darazam, I.A.; Shokouhi, S.; Pourhoseingholi, M.A.; Irvani, S.S.N.; Mokhtari, M.; Shabani, M.; Amirdosara, M.; Torabinavid, P.; Golmohammadi, M.; Hashemi, S.; et al. Role of interferon therapy in severe COVID-19: The COVIFERON randomized controlled trial. Sci. Rep. 2021, 11, 8059. [CrossRef]

95. Fang, J.; Li, H.; Du, W.; Yu, P.; Guan, Y.-Y.; Ma, S.-Y.; Liu, D.; Chen, W.; Shi, G.-C.; Bian, X.-L. Efficacy of Early Combination Therapy With Lianhuaqingwen and Arbidol in Moderate and Severe COVID-19 Patients: A Retrospective Cohort Study. Front. Pharmacol. 2020, 11, 560209. [CrossRef] [PubMed]

96. Wen, C.Y.; Xie, Z.W.; Li, Y.P.; Deng, X.L.; Chen, X.T.; Cao, Y.; Ou, X.; Lin, W.Y.; Li, F.; Cai, W.P.; et al. Real-world efficacy and safety of lopinavir/ritonavir and arbidol in treating with COVID-19: An observational cohort study. Zhonghua Nei Ke Za Zhi 2020, 59, E012. [CrossRef]

97. Deng, L.; Li, C.; Zeng, Q.; Liu, X.; Li, X.; Zhang, H.; Hong, Z.; Xia, J. Arbidol combined with LPV/r versus LPV/r alone against Corona Virus Disease 2019: A retrospective cohort study. J. Infect. 2020, 81, e1-e5. [CrossRef]

98. Chen, J.; Lin, S.; Niu, C.; Xiao, Q. Clinical evaluation of Shufeng Jiedu Capsules combined with umifenovir (Arbidol) in the treatment of common-type COVID-19: A retrospective study. Expert Rev. Respir. Med. 2020, 15, 257-265. [CrossRef] [PubMed]

99. Qu, X.K.; Hao, S.L.; Ma, J.H.; Wei, G.Y.; Song, K.Y.; Tang, C.; Gao, Y.F.; Liang, S.Q.; Du, W.J. Observation on clinical effect of Shufeng Jiedu Capsule combined with Arbidol Hydrochloride Capsule in treatment of COVID-19. Chin. Tradit. Herb. Drugs 2020, 51, 1167-1170. [CrossRef]

100. Yu, P.; Li, Y.Z.; Wan, S.B.; Wang, Y. Effects of Lianhua Qingwen Granules Plus Arbidol on Treatment of Mild Corona Virus Disease-19. Chin. Pharm. J. 2020, 55, 1042-1045. [CrossRef]

101. Xi, W.-N.; Jin, D.; Sun, K.; Yu, R.-Y.; Yao, X.-B.; Zou, B.-S.; Song, Z.-Y.; Yang, A.-Y.; Luo, R.-X.; Liu, Y.; et al. Treatment with Arbidol and Moxifloxacin in Ordinary and Severe Adult Patients Infected with COVID-19. medRxiv 2020. [CrossRef]

102. Huang, H.; Guan, L.; Yang, Y.; Le Grange, J.M.; Tang, G.; Xu, Y.; Yuan, J.; Lin, C.; Xue, M.; Zhang, X.; et al. Chloroquine, arbidol (umifenovir) or lopinavir/ritonavir as the antiviral monotherapy for COVID-19 patients: A retrospective cohort study. Res. Sq. 2020, 2020, 1-15. [CrossRef]

103. Xu, P.; Huang, J.; Fan, Z.; Huang, W.; Qi, M.; Lin, X.; Song, W.; Yi, L. Arbidol/IFN- $\alpha 2 b$ therapy for patients with corona virus disease 2019: A retrospective multicenter cohort study. Microbes Infect. 2020, 22, 200-205. [CrossRef]

104. Nojomi, M.; Yassin, Z.; Keyvani, H.; Makiani, M.J.; Roham, M.; Laali, A.; Dehghan, N.; Navaei, M.; Ranjbar, M. Effect of Arbidol (Umifenovir) on COVID-19: A randomized controlled trial. BMC Infect. Dis. 2020, 20, 954. [CrossRef] [PubMed]

105. Ghaderkhani, S.; Khaneshan, A.S.; Salami, A.; Alavijeh, P.E.; Kouchak, H.E.; Khalili, H.; Naghi, S.A.A.; Ahmadinejad, Z.; Rasolinejad, M.; Hajiabdolbaghi, M.; et al. Efficacy and Safety of Arbidol in Treatment of Patients with COVID-19 Infection: A Randomized Clinical Trial. Res. Sq. 2020, 2020, 1-13. [CrossRef]

106. Li, M.; Yu, T.; Zhu, J.; Wang, Y.; Yang, Y.; Zhao, K.; Yi, Y.; He, J.; Li, C.; He, J. Comparison of the antiviral effect of Arbidol and Chloroquine in treating COVID-19. Ann. Palliat. Med. 2021, 10, 3307-3312. [CrossRef]

107. Chiba, S. Effect of early oseltamivir on outpatients without hypoxia with suspected COVID-19. Wien. Klin. Wochenschr. 2020, 133, 292-297. [CrossRef] [PubMed]

108. Meriglier, E.; Rivoisy, C.; Hessamfar, M.; Bernard, N.; Aureau, I.; Lapoirie, J.; Contis, A.; Sacher, F.; Sacristan, B.; Lahouati, M.; et al. Safety of hydroxychloroquine and darunavir or lopinavir in COVID-19 infection. J. Antimicrob. Chemother. 2021, 76, 482-486. [CrossRef]

109. Davoudi-Monfared, E.; Rahmani, H.; Khalili, H.; Hajiabdolbaghi, M.; Salehi, M.; Abbasian, L.; Kazemzadeh, H.; Yekaninejad, M.S. A Randomized Clinical Trial of the Efficacy and Safety of Interferon $\beta-1$ a in Treatment of Severe COVID-19. Antimicrob. Agents Chemother. 2020, 64, e01061-20. [CrossRef]

110. Qu, J.; Li, G.-H.; Wang, J.-J.; He, G.-F.; Huang, J.-J.; Chen, Y.; Qu, Q.; Chen, X.-Y.; Lu, Q. Comparative effectiveness of Lopinavir/Ritonavir-based regimens in COVID-19. Clin. Exp. Pharmacol. Physiol. 2021, 48, 203-210. [CrossRef] [PubMed]

111. Joshi, S.; Parkar, J.; Ansari, A.; Vora, A.; Talwar, D.; Tiwaskar, M.; Patil, S.; Barkate, H. Role of FPVipiravir in the treatment of COVID-19. Int. J. Infect. Dis. 2021, 102, 501-508. [CrossRef]

112. Duyan, M.; Ozturan, I.U. Acute Psychosis in COVID-19: Is It Due to FPVipiravir Treatment or Acute Viral Illness? SN Compr. Clin. Med. 2021, 3, 1627-1629. [CrossRef]

113. Agrawal, U.; Raju, R.; Udwadia, Z.F. FPVipiravir: A new and emerging antiviral option in COVID-19. Med. J. Armed Forces India 2020, 76, 370-376. [CrossRef]

114. Kaur, R.J.; Charan, J.; Dutta, S.; Sharma, P.; Bhardwaj, P.; Sharma, P.; Lugova, H.; Krishnapillai, A.; Islam, S.; Haque, M.; et al. Favipiravir Use in COVID-19: Analysis of Suspected Adverse Drug Events Reported in the WHO Database. Infect. Drug Resist. 2020, 13, 4427-4438. [CrossRef]

115. De Andrea, M.; Ravera, R.; Gioia, D.; Gariglio, M.; Landolfo, S. The interferon system: An overview. Eur. J. Paediatr. Neurol. 2002, 6, A41-A46. [CrossRef]

116. Jakimovski, D.; Kolb, C.; Ramanathan, M.; Zivadinov, R.; Weinstock-Guttman, B. Interferon $\beta$ for Multiple Sclerosis. Cold Spring Harb. Perspect. Med. 2018, 8, a032003. [CrossRef] 
117. Hasselbalch, H.C.; Skov, V.; Kjær, L.; Ellervik, C.; Poulsen, A.; Poulsen, T.D.; Nielsen, C.H. COVID-19 as a mediator of interferon deficiency and hyperinflammation: Rationale for the use of JAK1/2 inhibitors in combination with interferon. Cytokine Growth Factor Rev. 2021, 60, 28-45. [CrossRef]

118. Yuan, J.; Zou, R.; Zeng, L.; Kou, S.; Lan, J.; Li, X.; Liang, Y.; Ding, X.; Tan, G.; Tang, S.; et al. The correlation between viral clearance and biochemical outcomes of 94 COVID-19 infected discharged patients. Inflamm. Res. 2020, 69, 599-606. [CrossRef] [PubMed]

119. Zhou, Q.; Chen, V.; Shannon, C.P.; Wei, X.-S.; Xiang, X.; Wang, X.; Wang, Z.-H.; Tebbutt, S.J.; Kollmann, T.R.; Fish, E.N. Interferon- $\alpha 2 b$ Treatment for COVID-19. Front. Immunol. 2020, 11, 1061. [CrossRef]

120. Wang, N.; Zhan, Y.; Zhu, L.; Hou, Z.; Liu, F.; Song, P.; Qiu, F.; Wang, X.; Zou, X.; Wan, D.; et al. Retrospective Multicenter Cohort Study Shows Early Interferon Therapy Is Associated with FPVorable Clinical Responses in COVID-19 Patients. Cell Host Microbe 2020, 28, 455-464.e2. [CrossRef]

121. Kaplan, S.S.; Hicks, C.B. Lopinavir/ritonavir in the treatment of human immunodeficiency virus infection. Expert Opin. Pharmacother. 2005, 6, 1573-1585. [CrossRef]

122. Podzamczer, D.; King, M.S.; Klein, C.E.; Flexner, C.; Katlama, C.; Havlir, D.V.; Letendre, S.L.; Eron, J.J.; Brun, S.C.; Bernstein, B. High-Dose Lopinavir/Ritonavir in Highly Treatment-Experienced HIV-1 Patients: Efficacy, Safety, and Predictors of Response. HIV Clin. Trials 2015, 8, 193-204. [CrossRef]

123. Meini, S.; Pagotto, A.; Longo, B.; Vendramin, I.; Pecori, D.; Tascini, C. Role of Lopinavir/Ritonavir in the Treatment of Covid-19: A Review of Current Evidence, Guideline Recommendations, and Perspectives. J. Clin. Med. 2020, 9, 2050. [CrossRef]

124. Choi, M.J.; Kang, M.; Shin, S.Y.; Noh, J.Y.; Cheong, H.J.; Kim, W.J.; Jung, J.; Song, J.Y. Comparison of antiviral effect for mild-tomoderate COVID-19 cases between lopinavir/ritonavir versus hydroxychloroquine: A nationwide propensity score-matched cohort study. Int. J. Infect. Dis. 2021, 102, 275-281. [CrossRef]

125. Yadollahzadeh, M.; Eskandari, M.; Roham, M.; Zamani, F.; Laali, A.; Yassin, Z.; Zeiaei, M.T.; Rahimian, N.; Moetamed, N.; Aliakbar, A.; et al. Evaluation of Sovodak (Sofosbuvir/Daclatasvir) Treatment Outcome in COVID-19 Patient's Compared with Kaletra (Lopinavir/ritonavir): A Randomized Clinical Trial. Res. Sq. 2021, 2021, 1-18. [CrossRef]

126. Hossain, M.J.; Rahman, S.M.A. Repurposing therapeutic agents against SARS-CoV-2 infection: Most promising and neoteric progress. Expert Rev. Anti. Infect. Ther. 2020, 19, 1009-1027. [CrossRef] [PubMed]

127. Wang, X.; Cao, R.; Zhang, H.; Liu, J.; Xu, M.; Hu, H.; Li, Y.; Zhao, L.; Li, W.; Sun, X.; et al. The anti-influenza virus drug, arbidol is an efficient inhibitor of SARS-CoV-2 in vitro. Cell Discov. 2020, 6, 1-5. [CrossRef] [PubMed]

128. Zhang, J.; Wang, W.; Peng, B.; Peng, W.; Zhang, Y.; Wang, Y.; Wan, Y.; Chang, J.; Mao, L.; Miao, X.; et al. Potential of Arbidol for Post-exposure Prophylaxis of COVID-19 Transmission: A Preliminary Report of a Retrospective Cohort Study. Curr. Med. Sci. 2020, 40, 480-485. [CrossRef] [PubMed]

129. Xu, K.; Chen, Y.; Yuan, J.; Yi, P.; Ding, C.; Wu, W.; Li, Y.; Ni, Q.; Zhou, R.; Li, X.; et al. Clinical Efficacy of Arbidol in Patients with 2019 Novel Coronavirus-Infected Pneumonia: A Retrospective Cohort Study. SSRN Electron. J. 2020, 2020, 1-28. [CrossRef]

130. Yadegarinia, D.; Tehrani, S.; Abolghasemi, S.; Zarghi, A.; Sali, S.; Zolfaghari, F. Evaluation of the Efficacy of Arbidol in Comparison with the Standard Treatment Regimen of Hospitalized Patients with Covid-19: A Randomized Clinical Trial. Arch. Clin. Infect. Dis. 2020, 15, 1-6. [CrossRef]

131. Chen, X.; Zhang, Y.; Zhu, B.; Zeng, J.; Hong, W.; He, X.; Chen, J.; Zheng, H.; Qiu, S.; Deng, Y.; et al. Associations of clinical characteristics and antiviral drugs with viral RNA clearance in patients with COVID-19 in Guangzhou, China: A retrospective cohort study. medRxiv 2020. [CrossRef]

132. McClellan, K.; Perry, C. Oseltamivir: A review of its use in influenza. Drugs 2001, 61, 263-283. [CrossRef] [PubMed]

133. Whitley, R.J.; Hayden, F.G.; Reisinger, K.S.; Young, N.; Dutkowski, R.; Ipe, D.; Mills, R.G.; Ward, P. Oral oseltamivir treatment of influenza in children. Pediatr. Infect. Dis. J. 2001, 20, 127-133. [CrossRef]

134. Wang, D.; Hu, B.; Hu, C.; Zhu, F.; Liu, X.; Zhang, J.; Wang, B.; Xiang, H.; Cheng, Z.; Xiong, Y.; et al. Clinical Characteristics of 138 Hospitalized Patients With 2019 Novel Coronavirus-Infected Pneumonia in Wuhan, China. JAMA 2020, 323, 1061-1069. [CrossRef]

135. Sanders, J.M.; Monogue, M.L.; Jodlowski, T.Z.; Cutrell, J.B. Pharmacologic Treatments for Coronavirus Disease 2019 (COVID-19): A Review. JAMA 2020, 323, 1824-1836. [CrossRef]

136. Wu, R.; Wang, L.; Kuo, H.-C.D.; Shannar, A.; Peter, R.; Chou, P.J.; Li, S.; Hudlikar, R.; Liu, X.; Liu, Z.; et al. An Update on Current Therapeutic Drugs Treating COVID-19. Curr. Pharmacol. Rep. 2020, 6, 56-70. [CrossRef]

137. Mancilla-Galindo, J.; García-Méndez, J.Ó.; Marquéz-Sánchez, J.; Reyes-Casarrubias, R.E.; Aguirre-Aguilar, E.; Rocha-González, H.I.; Kammar-García, A. All-cause mortality among patients treated with repurposed antivirals and antibiotics for COVID-19 in Mexico City: A real-world observational study. EXCLI J. 2021, 20, 199-222. [CrossRef]

138. Deeks, E.D. Darunavir/Cobicistat/Emtricitabine/Tenofovir Alafenamide: A Review in HIV-1 Infection. Drugs 2018, 78, 1013-1024. [CrossRef]

139. Chowdhury, K.H.; Chowdhury, R.; Mahmud, S.; Tareq, A.M.; Hanif, N.B.; Banu, N.; Reza, A.S.M.; Emran, T.B.; Simal-Gandara, J. Drug repurposing approach against novel coronavirus disease (COVID-19) through virtual screening targeting SARS-CoV-2 main protease. Biology 2021, 10, 2. [CrossRef]

140. Halder, U.C. Predicted antiviral drugs Darunavir, Indinavir and Rimantadine can potentially bind to neutralize COVID-19 conserved proteins. Res. Sq. 2020, 2020, 1-21. [CrossRef] 
141. OECD. Policy Responses to Coronavirus (COVID-19). Coronavirus (COVID-19) Vaccines for Developing Countries: An Equal Shot at Recovery. Available online: https://www.oecd.org/coronavirus/policy-responses/coronavirus-covid-19-vaccines-fordeveloping-countries-an-equal-shot-at-recovery-6b0771e6/ (accessed on 27 July 2021).

142. Bari, M.S.; Hossain, M.J.; Akhter, S.; Emran, T.B. Delta variant and black fungal invasion: A bidirectional assault might worsen the massive second/third stream of COVID-19 outbreak in South-Asia. Ethics Med. Public Health 2021, 19, 100722. [CrossRef]

143. Williamson, B.N.; Feldmann, F.; Schwarz, B.; Meade-White, K.; Porter, D.P.; Schulz, J.; Van Doremalen, N.; Leighton, I.; Yinda, C.K.; Pérez-Pérez, L.; et al. Clinical benefit of remdesivir in rhesus macaques infected with SARS-CoV-2. Nature 2020, 585, 273-276. [CrossRef] [PubMed]

144. Nagata, T.; Lefor, A.K.; Hasegawa, M.; Ishii, M. Favipiravir: A new medication for the Ebola virus disease pandemic. Dis. Med. Public Health Prep. 2015, 9, 79-81. [CrossRef]

145. Sharun, K.; Tiwari, R.; Dhama, K.; Emran, T.B.; Rabban, A.A.; Al Mutair, A. Emerging SARS-CoV-2 variants: Impact on vaccine efficacy and neutralizing antibodies. Hum. Vaccines Immunother. 2021, 17, 1-4. [CrossRef]

146. Hossain, M.J. Impact of COVID-19 pandemic among health care providers in Bangladesh: A systematic review. Bangladesh J. Infect. Dis. 2020, 7 (Suppl. S2), S8-S15. [CrossRef]

147. Hossain, M.J.; Islam, M.S.; Shahriar, S.; Sanam, S.; Emran, T.B.; Khatun, C.S.; Islam, M.R.; Mitra, S.; Dhama, K. Comedication of rabeprazole sodium causes potential drug-drug interaction with diabetic drug linagliptin: In-vitro and in-silico approaches. J. Exp. Biol. Agric. Sci. 2021, 9, 528-542. [CrossRef]

148. Lai, C.C.; Wang, C.Y.; Hsueh, P.R. Coinfections among patients with COVID-19: The need for combination therapy with non-anti-SARS-CoV-2 agents? J. Microbiol. Immunol. Infect. 2020, 53, 505-512. [CrossRef] [PubMed]

149. Zuo, Y.; Liu, Y.; Zhong, Q.; Zhang, K.; Xu, Y.; Wang, Z. Lopinavir/ritonavir and interferon combination therapy may help shorten the duration of viral shedding in patients with COVID-19: A retrospective study in two designated hospitals in Anhui, China. J. Med. Virol. 2020, 92, 2666-2674. [CrossRef] [PubMed] 